\title{
4 A discretization procedure for rare events in Bayesian networks
}

$5 \quad$ Kilian Zwirglmaier \& Daniel Straub

6 Engineering Risk Analysis Group, Technische Universität München

7 (kilian.zwirglmaier@tum.de, straub@tum.de,www.era.bgu.tum.de)

\section{Abstract}

9 Discrete Bayesian networks (BNs) can be effective for risk- and reliability assessments, in 10 which probability estimates of (rare) failure events are frequently updated with new 11 information. To solve such reliability problems accurately in BNs, the discretization of 12 continuous random variables must be performed carefully. To this end, we develop an 13 efficient discretization scheme, which is based on finding an optimal discretization for the 14 linear approximation of the reliability problem obtained from the First-Order Reliability 15 Method (FORM). Because the probability estimate should be accurate under all possible 16 future information scenarios, the discretization scheme is optimized with respected to the 17 expected posterior error. To simplify application of the method, we establish parametric 18 formulations for efficient discretization of random variables in BNs for reliability problems 19 based on numerical investigations. The procedure is implemented into a software prototype. 20 Finally, it is applied to a verification example and an application example, the prediction of 21 runway overrun of a landing aircraft.

\section{Keywords}

23 Bayesian networks; discretization; near-real-time; structural reliability; updating 


\section{Introduction}

25 For operational risk and reliability management, it is often desirable to compute the probability of a rare event $F$ under potentially evolving information. Examples include warning systems for natural and technical hazards, or the planning of inspection and intervention actions in infrastructure systems. Ideally, this is achieved through Bayesian updating of $\operatorname{Pr}(F)$ with the new information $Z$ to the posterior probability $\operatorname{Pr}(F \mid Z)$. When physically-based or empirical models for predicting the rare event exist, such updating is possible with structural reliability methods (SRM) (Sindel and Rackwitz, 1998, Straub, 2011, Straub et al., 2016). However, it is often difficult to perform the required computations in near-real-time, due to a lack of efficiency or robustness. A modeling and computational framework that does facilitate efficient Bayesian updating is the discrete Bayesian network $(\mathrm{BN})$. Hence it was proposed to combine SRMs with discrete Bayesian networks for nearreal-time computations (Friis-Hansen, 2000, Straub and Der Kiureghian, 2010a, Straub and Der Kiureghian, 2010b).

BNs are based on directed acyclic graphs (DAGs), to efficiently define a joint probability distribution $p(\mathbf{Y})$ over a random vector $\mathbf{Y}$ (Jensen and Nielsen, 2007, Kjaerulff and Madsen, 2013). The DAG of a BN, which is often referred to as the qualitative part of a BN, consists of a node for each variable in $\mathbf{Y}$ and a set of directed links among nodes representing dependence among the variables. In the case of discrete BNs, conditional probability tables (CPTs) quantitatively define the type and strength of the dependence among the variables. The entries of the CPT of a variable $Y_{i}$ are the probabilities for each state of $Y_{i}$ conditional on all possible combinations of states of its parents.

For hybrid BNs, which include both discrete and continuous variables, exact inference is available only for two special cases, which are BNs with Gaussian nodes, whose means are linear functions of their parents, and BNs, whose nodes are defined as a mixture of truncated basic functions (MoTBFs) (Langseth et al., 2009, Langseth et al., 2012). Otherwise, approximate inference algorithms are available for hybrid BNs based on sampling techniques, e.g. (Lerner, 2002, Hanea et al., 2006). However, these are computationally demanding and not generally suitable for near-real-time decision support (Hanea et al., 2015). As an alternative, the continuous random variables can be discretized, which enables the use of exact inference algorithms that exist for general discrete BNs. These include the variable elimination algorithm (Zhang and Poole, 1994) and the junction tree algorithm (Lauritzen and Spiegelhalter, 1988, Jensen et al., 1990).

The size of discrete BNs, and the associated computational effort, increases approximately exponentially with the number of discrete states of its nodes, which motivates the development of efficient discretization algorithms. While efficient discretization in the context of machine learning and BNs in general has been investigated by multiple researchers (Dougherty et al., 1995, Kotsiantis and Kanellopoulos, 2006), research on efficient discretization in the context of engineering risk analysis or structural reliability has been 
the former discretizes the BN a-priori before entering evidence (offline), the latter is based on an iterative scheme that updates the discretization scheme in function of the evidence (online).

Dynamic discretization for risk analysis applications has been developed mainly by (Neil et al., 2008), based on the work by (Kozlov and Koller, 1997). The procedure starts with an initial discretization of a hybrid $\mathrm{BN}$, for which an approximate entropy error is calculated. If the error complies with a convergence criterion, the current discretization is accepted. Otherwise the discretization is iteratively altered, by splitting the intervals with the highest entropy error, until the convergence criterion is fulfilled. The approach is implemented in the software AgenaRisk (Agena, 2005). Other dynamic discretization algorithms for reliability analysis have been proposed, e.g. in (Zhu and Collette, 2015) for dynamic BNs. The advantage of dynamic discretization is its flexibility when evidence is entered in the BN, i.e. when the model is updated with new observation.

Static discretization has the advantage of being computationally faster and simple to implement. Some considerations for static discretization of BNs in reliability applications have been presented in (Friis-Hansen, 2000, Straub, 2009, Straub and Der Kiureghian, 2010a). As pointed out by (Friis-Hansen, 2000), for applications in which extreme events are important, discretization of the distribution tails should be performed with care. Static discretization facilitates a careful representation of these tails. However, the accuracy of the static discretization varies with the available evidence. The difficulty is thus to find a discretization scheme that is optimal under a wide variety of posterior distributions.

In this paper we derive a procedure for efficiently performing static discretization of continuous reliability problems. An optimal discretization scheme is sought, which minimizes the expected approximation error with respect to possible future observations (evidence). To solve this optimization problem, we propose to approximate the reliability problem by the First-Order Reliability Method (FORM). Section 2 of the paper describes the proposed methodology. Section 3 presents numerical parameter studies, and simple parametric relations for defining an efficient discretization scheme are derived. In Section 4, the procedure is applied to a set of verification examples and to the computation of the probability of runway overrun of a landing aircraft. While the theory is introduced for problems with only one design point, considerations regarding problems with multiple design points are given in the last verification example and in the discussion.

\section{Methodology}

\subsection{Structural reliability}

Since the 1970s structural reliability methods have been developed and applied in the engineering community to estimate failure probabilities $\operatorname{Pr}(F)$ of components or systems, based on physical or empirical models. The performance of engineering components is 
described by a limit state function (LSF) $g(\mathbf{x})$, where $\mathbf{X}=\left[X_{1} ; \ldots ; X_{n}\right]$ is a vector of basic random variables influencing the performance of the component. By definition, failure corresponds to $g(\mathbf{x})$ taking non-positive values, i.e. the failure event is $F=\{g(\mathbf{X}) \leq 0\} . g(\mathbf{x})$ includes the physical or engineering model, which is often computationally demanding. The

104 probability of failure is calculated by integrating the probability density function (PDF) of $\mathbf{X}$,

$105 f_{\mathbf{X}}(\mathbf{x})$, over the failure domain:

$\operatorname{Pr}(F)=\int_{g(\mathbf{x}) \leq 0} f_{\mathbf{X}}(\mathbf{x}) d \mathbf{x}$

106 The formulation can be extended to the reliability of general systems by defining the failure 107 domain as a combination of series and parallel systems (Ditlevsen and Madsen, 2007). In the 108 general case, there is no analytical solution to Eq. 2 and the integral is potentially high109 dimensional. For this reason, structural reliability methods (SRMs) are applied to approximate 110 it. These include the first- and the second order reliability method (FORM and SORM) as 111 well as a large variety of sampling methods, including importance sampling methods such as 112 directional importance sampling, and sequential sampling methods such as subset simulation. 113 These methods are well-documented in the literature (Au and Beck, 2001, Rackwitz, 2001, 114 Der Kiureghian, 2005, Ditlevsen and Madsen, 2007).

\section{$115 \quad 2.2$ First order reliability method (FORM)}

116 To obtain an approximation of the probability of failure through FORM, the LSF $g(\mathbf{X})$ is 117 transformed to an equivalent $\operatorname{LSF} G(\mathbf{U})$ in the space of uncorrelated standard normal random 118 variables $\mathbf{U}=\left[U_{1} ; \ldots ; U_{n}\right]$ (Fig. 1). The transformation is probability conserving, so that $119 \operatorname{Pr}[g(\mathbf{X}) \leq 0]=\operatorname{Pr}[G(\mathbf{U}) \leq 0]=\operatorname{Pr}(F)$. A suitable transformation for this purpose, which is 120 consistent with the BN, is the Rosenblatt transformation (Hohenbichler and Rackwitz, 1981).

121 In case all basic random variables are independent, this transformation reduces to the 122 marginal transformations: $U_{i}=\Phi^{-1}\left[F_{X_{i}}\left(X_{i}\right)\right]$, with $\Phi^{-1}$ being the inverse standard normal 123 CDF. 
(a) original variable space

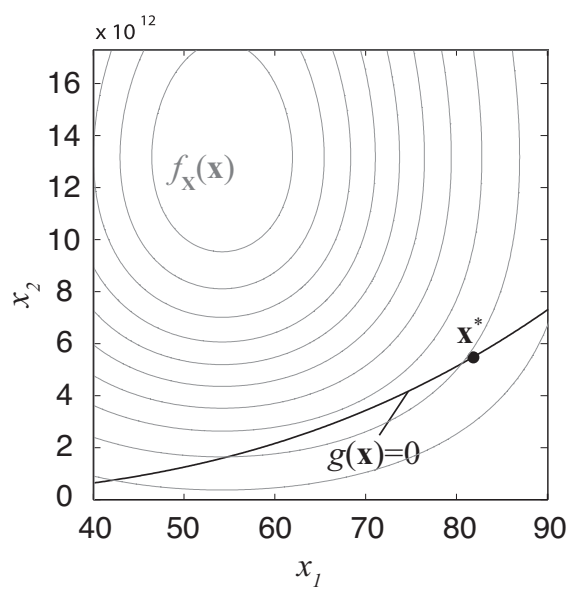

(b) standard normal space
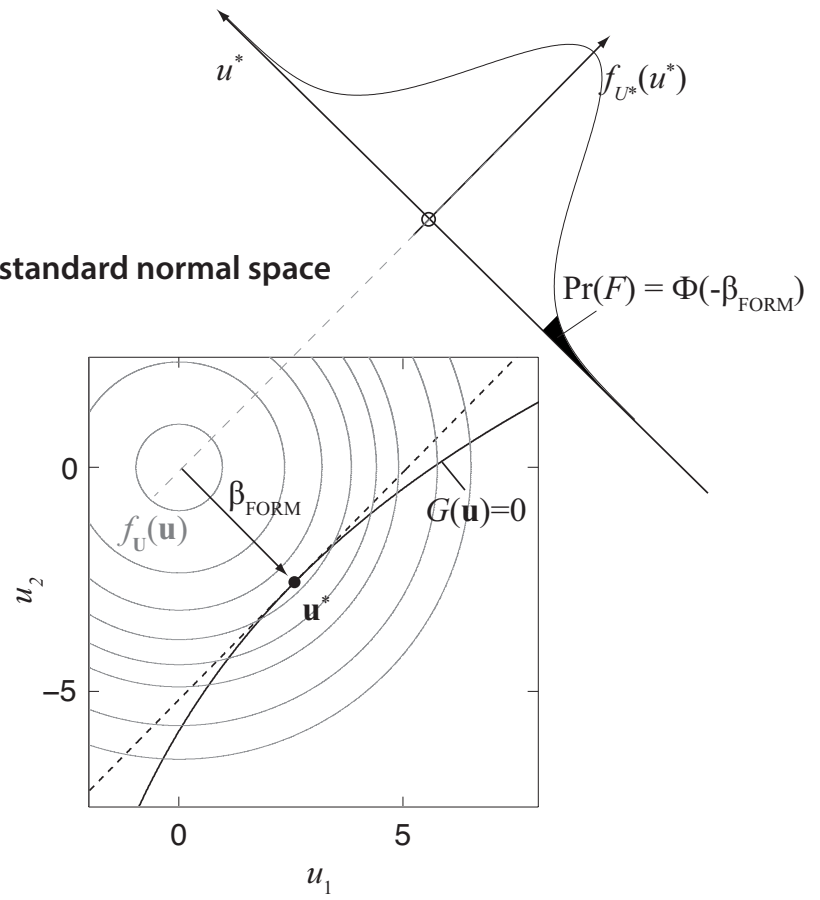

124

125

126

127

128

129

130

131

132

133

134

$$
\operatorname{Pr}\left[G_{L}(\mathbf{U}) \leq 0\right]=\Phi\left(-\beta_{F O R M}\right)
$$

135 where $\Phi$ is the standard normal $\mathrm{CDF}$ and $\beta_{F O R M}$ is the distance from the origin to the design 136 point, i.e. $\beta_{F O R M}=\left\|\mathbf{u}^{*}\right\|$. The problem thus reduces to finding the design point $\mathbf{u}^{*}$. If $G(\mathbf{U})$ is 137 linear, the FORM solution of the probability of failure is exact, otherwise it is an 138 approximation, which however is sufficiently accurate in most practical applications with 139 limited numbers of random variables (Rackwitz, 2001).

140 The linearized LSF $G_{L}(\mathbf{U})$ can be written as:

$G_{L}(\mathbf{U})=\beta_{F O R M}-\alpha^{\mathrm{T}} \mathbf{U}$

141 where $\boldsymbol{\alpha}=\left[\alpha_{1}, \ldots, \alpha_{n}\right]$ is the vector of FORM importance measures. These importance 142 measures are defined as:

$$
\alpha_{i}=\frac{u_{i}^{*}}{\beta_{F O R M}}
$$


where $u_{i}^{*}$ is the $i$-th component of the design point coordinates. The $\alpha_{i}$ 's take values between -1 and 1 , and it is $\|\boldsymbol{\alpha}\|=1 . \alpha_{i}$ is 0 , if the uncertainty on $U_{i}$ has no influence on $\operatorname{Pr}\left(G_{L}(\mathbf{U}) \leq\right.$ 0 ), and it is 1 or -1 , if $U_{i}$ is the only random variable affecting $\operatorname{Pr}\left(g_{L}(\mathbf{U}) \leq 0\right)$. When the original random variables $X_{i}$ are mutually independent, the $\alpha_{i}$ 's are readily applicable also in the original space, otherwise the $\alpha_{i}$ 's can be transformed as described in (Der Kiureghian, 2005).

\subsection{Treatment of a reliability problem in a $\mathrm{BN}$}

We combine discrete BNs and structural reliability concepts to facilitate updating of rare event (failure) probabilities under new observations. The general problem setting is illustrated in the BN of Fig. 2. We here limit the presentation to component reliability problems; system problems are considered later. The binary random variable 'Component performance' is described by the LSF $g(\mathbf{X})$.

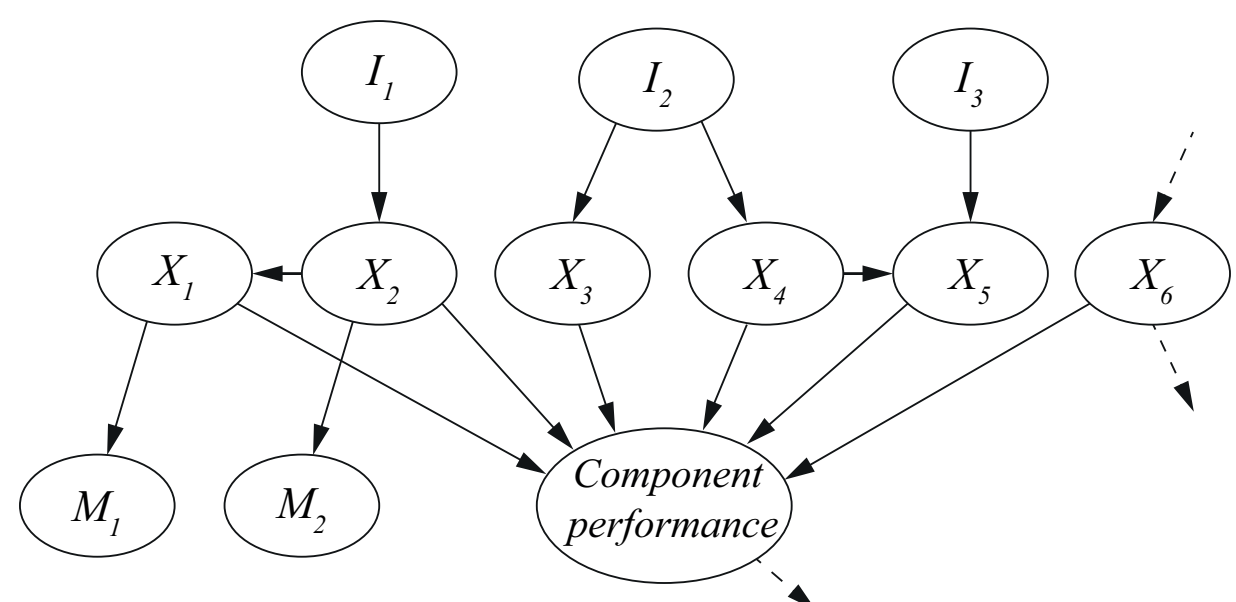

Figure 2. A general BN including a component reliability problem.

\section{8}

The basic random variables $\mathbf{X}$ of the model are included in the BN as parents of 'Component performance'. The nodes $M_{i}$ represent measurements of individual random variables $X_{i}$, and nodes $I_{j}$ represent factors influencing the basic random variables. Dependence between the variables in $\mathbf{X}$ is modeled either directly by links among them (here $X_{2} \rightarrow X_{1}$ and $X_{4} \rightarrow X_{5}$ ) or through common influencing factors (here $I_{2} \rightarrow X_{3}$ and $I_{2} \rightarrow X_{4}$ ). The component performance node can have (multiple) child nodes, which, however, does not impact the discretization of the reliability problem.

Ultimately, the goal is to predict the component performance, i.e. $\operatorname{Pr}(F)$, conditional on other model parameters, such as measurements $M_{i}$ or influencing variables $I_{j}$. Whenever new evidence on these variables is available, the $\mathrm{BN}$ should be evaluated (in near-real time) utilizing exact BN inference algorithms.

To enable exact inference algorithms, all continuous random variables are discretized. These include the $\mathbf{X}$, and possibily the $M_{i}$ and $I_{j}$. In the general case, the computational effort for solving the BN is a direct function of the CPT size of 'Component performance'. The size of 
this CPT is $2 \prod_{i=1}^{n} n_{i}$, where $n$ is the number of random variables in $\mathbf{X}$, and $n_{i}$ is the number of states used for discretizing $X_{i}$. In this paper we do not describe the discretization of random variables $M_{i}$ and $I_{j}$, since it is typically straightforward and does not contribute significantly to computational performance. The key parameter for computational efficiency and accuracy is the discretization scheme for $\mathbf{X}$, which is described in sections 2.5 and 2.6.

\section{7}

178

179

180

181

182

183

184

185

186

187

188

190

191

192

193

194

195

196

$$
\sigma_{G_{L}}^{2}=\|\nabla G\|^{2}\left(\alpha_{1}^{2}+\alpha_{2}^{2}+\cdots+\alpha_{n}^{2}\right)
$$

197 where $\nabla G$ denotes the gradient vector of the non-linearized LSF $G(\mathbf{U})$. From Eq. 6 it is seen 198 that a random variable $X_{i}$ with corresponding $\alpha_{i}$ accounts for $\alpha_{i}^{2} \cdot 100 \%$ of the variance $\sigma_{G_{L}}^{2}$. 199 Therefore, observing a random variable $X_{i}$ with $\alpha_{i}=0.1$ will reduce the variance $\sigma_{G_{L}}^{2}$ by $1 \%$, 200 whereas observing $X_{j}$ with $\alpha_{j}=0.5$ will reduce $\sigma_{G_{L}}^{2}$ by $25 \%$.

\subsection{Discretization of basic random variables}

202 For ease of presentation, we first consider discretization of statistically independent basic 203 random variables $\mathbf{X}$, i.e. the special case of the BN in Fig. 2 in which the $X_{i}$ 's have no parents ${ }^{1}$.

\footnotetext{
${ }^{1}$ In a BN, basic random variables $\mathbf{X}$ are independent if they are not connected through links, if they have no common (unknown) ancestors and if no evidence is available on any of their joint descendants.
} 
The proposed procedure is extended to the general case of dependent basic random variables thereafter.

\subsubsection{Independent basic random variables}

The situation is illustrated in Fig. 3. The performance of the component depends on $n$ statistically independent random variables and is described by a LSF $g(\mathbf{X})=g\left(X_{1}, \ldots, X_{n}\right)$. For all basic random variables $X_{i}$, corresponding measurements $M_{i}$ can be performed. To obtain an equivalent discrete $\mathrm{BN}$, the continuous $X_{i}$ are replaced by the discrete random variables $Y_{i}$, and the LSF is replaced by the CPT of component performance conditional on $\mathbf{Y}=\left[Y_{1} ; \ldots ; Y_{n}\right]$. For each discrete random variable $Y_{i}$ with $n_{i}$ states $1,2, \ldots, n_{i}$, we define a discretization scheme $D_{i}=\left[d_{0}, d_{1}, \ldots, d_{n_{i}-1}, d_{n_{i}}\right]$ consisting of $n_{i}+1$ interval boundaries. The first and the last interval boundaries are given by the boundaries of $X_{i}{ }^{\prime} s$ outcome space.

a) BN with continuous nodes

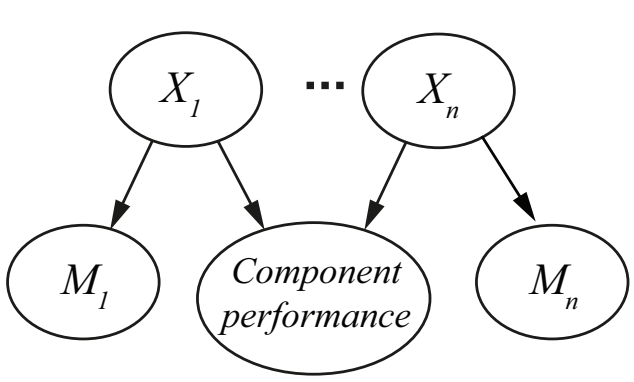

discretization: replace $X_{i}$ with $Y_{i}$

Figure 3. Representation of a basic reliability problem with $n$ independent basic random variables in a BN. Left: original problem with continuous basic random variables $X_{i}$, right: discrete $\mathrm{BN}$, in which $X_{i} \mathrm{~s}$ are substituted with discrete nodes $Y_{i}$.

220 Since here the $X_{i}$, and thus the $Y_{i}$, have no parents, the PMF of $Y_{i}$ is defined as:

$$
p_{Y_{i}}(j)=F_{X_{i}}\left(d_{j}\right)-F_{X_{i}}\left(d_{j-1}\right) ; \quad \text { with } j \epsilon\left[1, \ldots, n_{i}\right]
$$

where $F_{X_{i}}$ denotes the cumulative distribution function $(\mathrm{CDF})$ of $X_{i}$. The probability of failure corresponding to the discrete $\mathrm{BN}$ in Fig. $3 \mathrm{~b}$ can be calculated as:

$\operatorname{Pr}(F)=\sum_{y_{1}=1}^{n_{1}} \ldots \sum_{y_{n}=1}^{n_{n}} p_{Y_{1}}\left(y_{1}\right) \cdot \ldots \cdot p_{\mathrm{Y}_{n}}\left(y_{n}\right) \cdot \operatorname{Pr}\left(F \mid Y_{1}=y_{1} \cap \ldots \cap Y_{n}=y_{n}\right)$

223 Note that the discretization does not introduce any approximation error here, as long as the conditional $\operatorname{Pr}\left(F \mid Y_{1}=y_{1} \cap \ldots \cap Y_{n}=y_{n}\right)$ is computed exactly.

225 Once measurements from the nodes $\mathbf{M}=\left[M_{1} ; \ldots ; M_{n}\right]$ are available, the conditional failure 226 probability can be calculated as:

$$
\operatorname{Pr}(F \mid \mathbf{M}=\mathbf{m}) \approx \frac{1}{p_{\mathbf{M}}(\mathbf{m})} \sum_{y_{1}=1}^{n_{1}} \ldots \sum_{y_{\mathrm{n}}=1}^{n_{n}} \begin{gathered}
p_{Y_{1}}\left(\mathrm{y}_{1}\right) \cdot p_{M_{1} \mid Y_{1}}\left(m_{1} \mid y_{1}\right) \cdot \ldots \cdot p_{Y_{n}}\left(y_{n}\right) \cdot p_{M_{n} \mid Y_{n}}\left(m_{n} \mid y_{n}\right) \\
\cdot \operatorname{Pr}\left(F \mid Y_{1}=y_{1} \cap \ldots \cap Y_{n}=y_{n}\right)
\end{gathered}
$$


where $\operatorname{Pr}\left(F \mid Y_{1}=y_{1}, \ldots, Y_{n}=y_{n}\right)$ is the conditional probability of component failure given $\mathrm{y}_{1}, \ldots, \mathrm{y}_{\mathrm{n}}$. If no measurements are available for some of the basic random variables, the corresponding likelihood terms $\mathrm{p}_{\mathrm{M}_{\mathrm{j}} \mid \mathrm{Y}_{\mathrm{j}}}\left(\mathrm{m}_{\mathrm{j}} \mid \mathrm{y}_{\mathrm{j}}\right)$ are simply omitted in Eq. 9.

230 While the computation of the unconditional failure probability following Eq. 8 is exact, the 231 computation of the conditional failure probability through Eq. 9 is only an approximation. The reason is that the dependence between the measurement variable $M_{i}$ and the 'Component performance' variable is not fully captured in the discrete BN (see also Straub and Der

234 Kiureghian, 2010b). In Fig. 4, this is illustrated for a reliability problem with one basic 235 random variable $X_{i}$. Both the continuous distribution (Fig. 4a) and the corresponding 236 discretized distribution (Fig. 4b) are updated correctly after observing $M_{1}$. However, for Eq. 9 237 to be exact, also the conditional failure probabilities $\operatorname{Pr}\left(F \mid Y_{1}=y_{1}\right)$ would need to be updated. 238 This can be observed in Fig. 4a: in interval $Y_{1}=3$, which is the one cut by the limit state 239 surface, the ratio of the probability mass in the failure domain to that in the safe domain 240 changes from the prior to the posterior case, i.e. $\operatorname{Pr}\left(F \mid Y_{1}=3\right) \neq \operatorname{Pr}\left(F \mid Y_{1}=3, M_{1}=m_{1}\right)$. 241 Since the computation of the conditional failure probability following Eq. 9 is based on the 242 prior probability $\operatorname{Pr}\left(F \mid Y_{1}=3\right)$, the discretization introduces an approximation in this case. 243 The error occurs only in the intervals that are cut by the limit state surface.

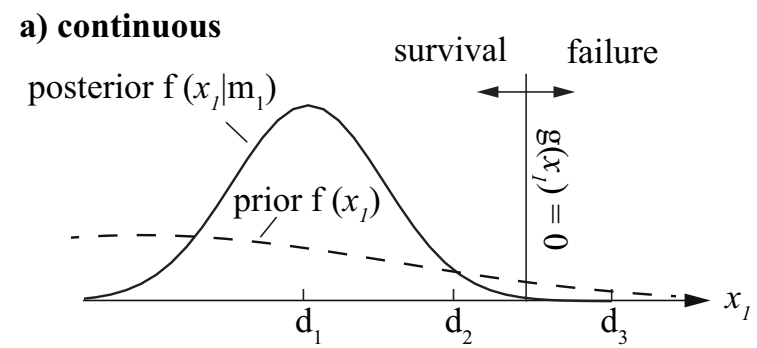

b) discrete

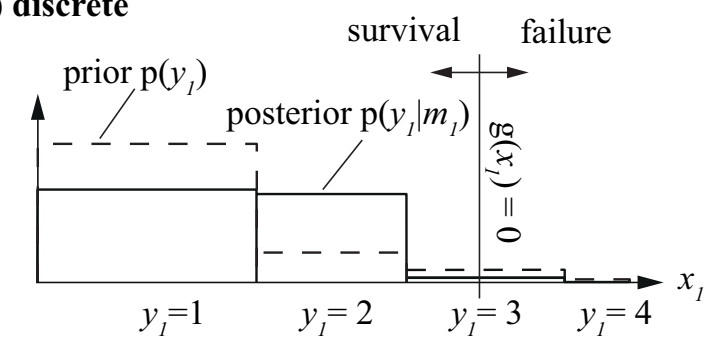

Figure 4. Discretization error in 1D.

247 In the simple one-dimensional case of Fig. 4, an optimal discretization approach would be to 248 discretize the whole outcome space in two intervals, one capturing the survival and one the 249 failure domain. This discretization would have zero approximation error. However, already in 250 a two-dimensional case, such a solution is not possible. This is illustrated in Fig. 5, where the cells cut by the limit state surface are indicated in grey. The failure probability conditional on measurements calculated according to Eq. 9 will necessarily be an approximation. The approximation error will be small, if the contribution of the cells cut by the limit state surface 


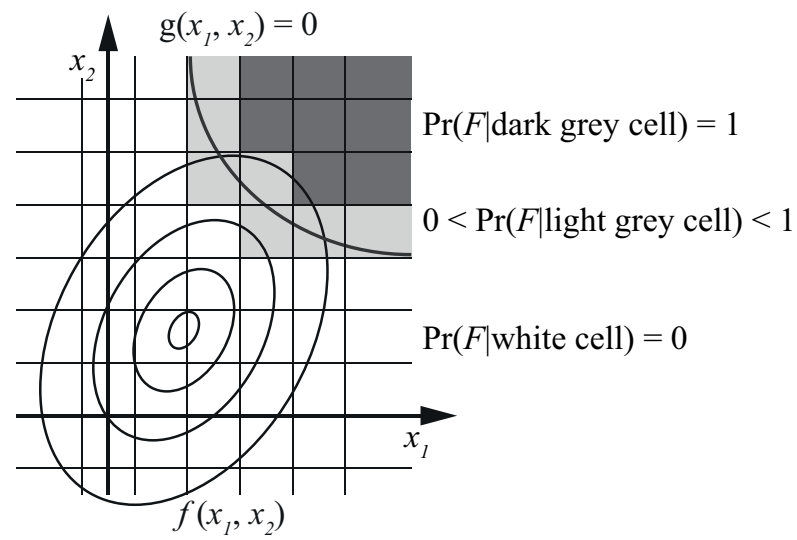

Figure 5. Discretization error in 2D.

\subsubsection{Dependent basic random variables}

259

260

261

262

263

264

265

Eqs. (7-9) must be adjusted when dependence among the $X_{i}$ 's is present, in accordance with the case-specific BN structure. However, the principles outlined above for independent $X_{1}, \ldots, X_{n}$ hold equally for dependent basic random variables: The discretization error is a function of the cells cut by the limit state function.

When determining an optimal discretization, we propose in the following to find the FORM approximation of the reliability problem, which can readily account for the dependence among the random variables. Hence, there is no need to distinguish between cases with independent or dependent random variables.

\subsection{Efficient discretization}

\subsubsection{Optimal discretization of linear problems in standard normal space}

To find an efficient discretization of $\mathbf{X}$, we consider the FORM solution to the reliability problem. Evaluating the linearized FORM LSF $G_{L}(\mathbf{U})$ is computationally inexpensive once the design point $\mathbf{u}^{*}$ is known. Therefore, it is feasible to find a discretization of $\mathbf{U}$ that is optimal for the event $\left\{G_{L}(\mathbf{U}) \leq 0\right\}$ through optimization. If $G(\mathbf{U})$ is not strongly non-linear, this solution will be an efficient discretization for $\{G(\mathbf{U}) \leq 0\}$ and, after a transformation to the original space, also for $\{g(\mathbf{X}) \leq 0\}$.

As discussed in Section 2.5.1, the approximation error of the discretization is associated with the change from the prior to the posterior distribution of the basic random variables. A measure of optimality must thus consider possible measurements of $\mathbf{X}$ or $\mathbf{U}$. We consider hypothetical measurements $\widetilde{M}_{i}$ (this notation is used to distinguish hypothetical measurements from actual measurements $M_{i}=m_{i}$ ) of all standard normal random variables $U_{i}$ with additive measurement error $\varepsilon_{\mathrm{i}} \sim N\left(0, \sigma_{\varepsilon_{i}}\right)$. Since both the prior distribution and the measurement error are normal distributed, the likelihood is also normal distributed: 


$$
\widetilde{M}_{i} \mid\left\{U_{i}=u_{i}\right\} \sim N\left(u_{i}, \sigma_{\varepsilon_{i}}\right)
$$

282 The posterior, i.e. the conditional distribution of $U_{i}$ given a measurement outcome $\widetilde{M}_{i}=\widetilde{m}_{i}$, is the normal distribution with mean $\frac{1}{1+\sigma_{\varepsilon_{i}}^{2}} \widetilde{m}_{i}$ and standard deviation $\sqrt{\left(1-\frac{1}{1+\sigma_{\varepsilon_{i}}^{2}}\right)}$.

284

We define an error measure based on comparing the true posterior probability of failure $\mathrm{P}_{\mathrm{F} \mid \widetilde{\boldsymbol{M}}}(\widetilde{\mathbf{m}})$ with the posterior probability of failure calculated from the discretized $\mathbf{U}$, denoted by $\widehat{\mathrm{P}}_{\mathrm{F} \mid \widetilde{\boldsymbol{M}}}(\mathbf{d} ; \widetilde{\mathbf{m}})$. Here, $\mathbf{d}$ are the parameters defining the discretization. The proposed error measure is:

$$
e(\mathbf{d}, \widetilde{\mathbf{m}})=\left|\frac{\log _{10} \widehat{\mathrm{P}}_{\mathrm{F} \mid \widetilde{M}}(\mathbf{d} ; \widetilde{\mathbf{m}})-\log _{10} \mathrm{P}_{\mathrm{F} \mid \widetilde{M}}(\widetilde{\mathbf{m}})}{\log _{10} \mathrm{P}_{\mathrm{F} \mid \widetilde{M}}(\widetilde{\mathbf{m}})}\right|
$$

The error measure of Eq. 11 represents a tradeoff between the absolute and the relative error. It weights the (logarithmic) relative error by the magnitude of the posterior failure probability. This ensures that the same relative error is considered worse at a higher probability level compared to an error at a lower probability level.

A-priori, the measurement outcomes are not known. Hence we define the optimal discretization as the one that minimizes the expected preposterior error $\mathrm{E}_{\widetilde{M}}[e(\mathbf{d}, \widetilde{\boldsymbol{M}})]$ :

$$
\mathbf{d}^{\text {opt }}=\arg \min _{\mathbf{d}} \mathrm{E}_{\widetilde{M}}[e(\mathbf{d}, \widetilde{\boldsymbol{M}})]=\arg \min _{\mathbf{d}} \int_{\widetilde{\boldsymbol{M}}} e(\mathbf{d}, \widetilde{\mathbf{m}}) \mathrm{f}_{\widetilde{M}}(\widetilde{\mathbf{m}}) \mathrm{d} \widetilde{\mathbf{m}}
$$

The optimization is thus based on the computation of an expected value with respect to the possible measurements outcomes $\widetilde{\boldsymbol{M}}$ before having taken any measurements. This is analogous to a preposterior analysis (Raiffa and Schlaifer, 1961, Straub, 2014b). However, unlike in traditional preposterior analysis, the objective is not to identify an optimal action under future available information, but to find the optimal discretization parameters $\mathbf{d}^{\text {opt }}$. The integral in Eq. 12 is evaluated through a simple Monte Carlo approach. All $\widetilde{M}_{i}$ have the normal distribution with zero mean and variance $1+\sigma_{\epsilon}^{2}$.

The parameters in $\mathbf{d}$ describing the discretization scheme are:

- $n_{\mathrm{i}}$ : number of intervals used to discretize each random variable $U_{i}$,

- $w_{\mathrm{i}}$ : width of the discretization frame in the dimension of $U_{i}$, and

- $v_{i}$ : position of the midpoint of the discretization frame relative to the design point

These parameters are illustrated in Fig. 6. For a problem with $n$ basic random variables, the full set of optimization parameters is $\mathbf{d}=\left[w_{1}, \ldots, w_{n}, n_{1}, \ldots, n_{n-1}, v_{1}, \ldots, v_{n}\right]$.

Clearly, the discretization error reduces with increasing $n_{i}$. Because the computational efficiency of the final $\mathrm{BN}$ is a direct function of the size of the CPT associated with component performance, which is $\prod_{i=1}^{n} n_{i}$, we constrain its size. To this end, we define $c_{u p}$ as 


$$
\prod_{i=1}^{n} n_{i} \leq c_{u p}
$$

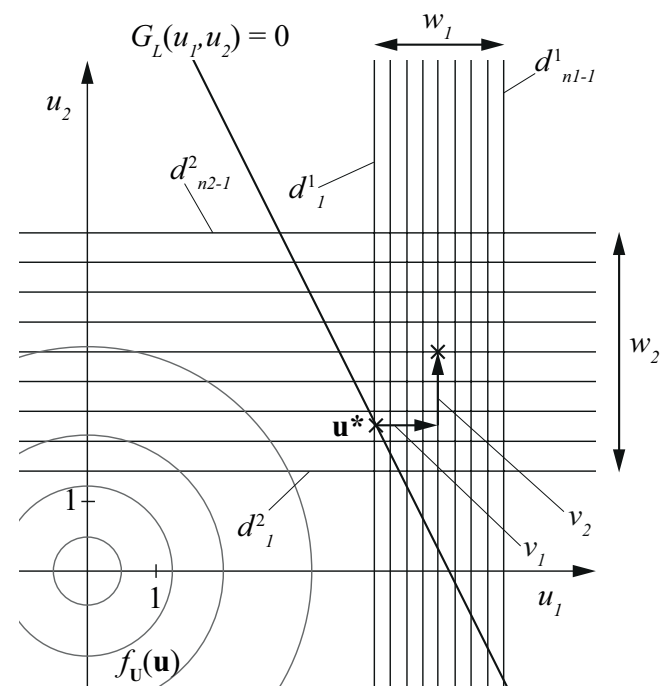

Figure 6. Schematic representation of a discretization of a linear 2D reliability problem. $w_{i}$ is the distance between interval boundaries $d_{1}^{i}$ and $d_{n_{i}-1}^{i}$. All intervals between these boundaries are equi-spaced. $v_{i}$ is the position of the midpoint of the discretization frame relative to the design point $\mathbf{u}^{*}$ in dimension $i$.

The optimization is implemented through a two-level approach. The optimization of the continuous parameters width $w_{i}$ and position of the discretization frame $v_{i}$ for all $i=1, \ldots, n$ is carried out using unconstrained nonlinear optimization for fixed values of $n_{i}$. The optimization of the discrete $n_{i}$ is performed through a local search algorithm. Note that the optimization is performed offline, i.e. prior to running the $\mathrm{BN}$, hence it does not affect the goal of near-real time performance of the BN. Furthermore, in section 3 a heuristic is derived that can replace the time-consuming solution of the optimization problem.

\subsubsection{Efficient discretization of the original random variables $\mathbf{X}$}

324 Since the nodes in the BN represent random variables $\mathrm{X}$ in their original outcome space, the discretization schemes, which are derived for the corresponding standard normal random variables $U$, need to be transformed to the X-space. In the case of mutually independent random variables $X_{i}$, any point on the $i$-th interval boundary in $U$-space - if transformed - will result in the same corresponding $\mathrm{i}$-th interval boundary in $\mathrm{X}$-space. This is not the case for dependent random variables $\mathrm{X}_{\mathrm{i}}$, where a mapping of the interval boundaries in U-space to $\mathrm{X}$ space will not lead to an orthogonal discretization scheme in $\mathrm{X}$-space. To preserve orthogonality throughout the transformation, we propose to represent each interval boundary through a characteristic point and determine the boundary in X-space through a transformation of this point. For transforming the interval boundary of $\mathrm{X}_{\mathrm{i}}$, the characteristic 
point is selected as the design point $\mathrm{u}^{*}$, where the $\mathrm{i}$-th element is substituted by the coordinate of the interval boundary. In Fig. 7 this is shown for an example with $n=2$ random variables.

a) Discretization in U-space

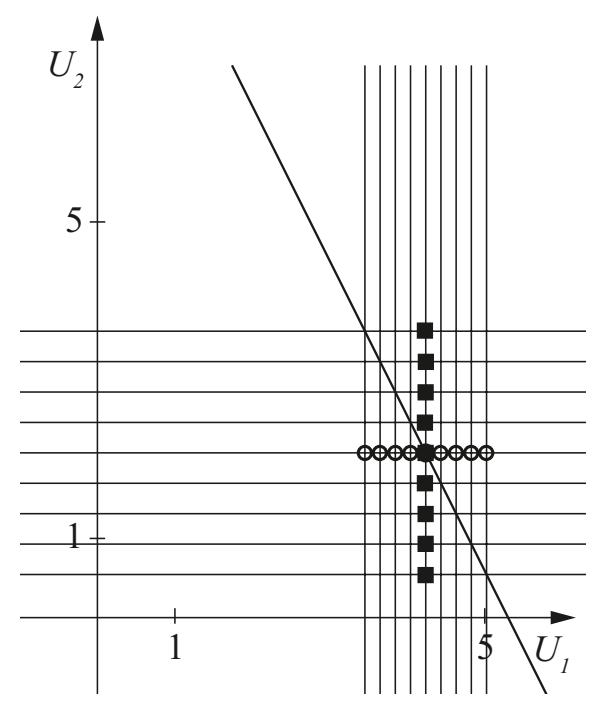

b) Transformed discretization

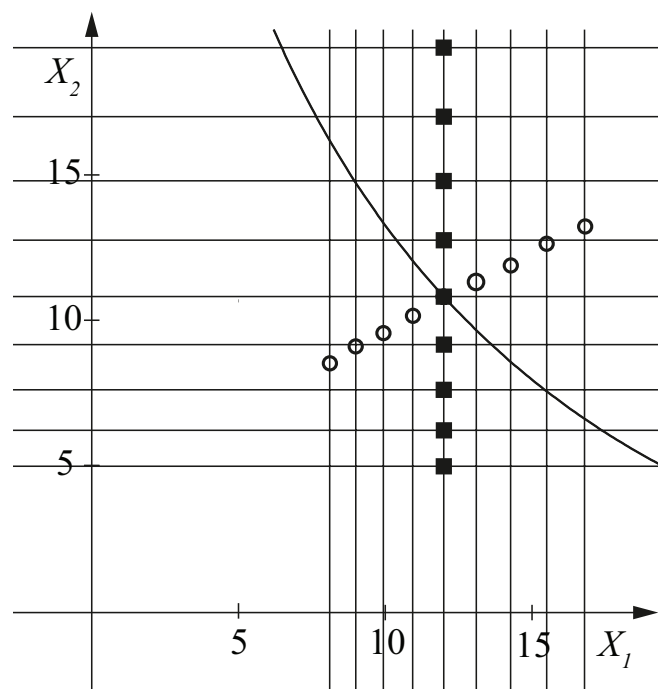

Figure 7. Transformation of a discretization scheme from U-space to X-space. To preserve orthogonality each interval boundary in U-space is represented by a characteristic point. The random variables $X_{1}$ and $X_{2}$ are Weibull distributed with scale and shape parameter 1 and their correlation is 0.5 .

\section{Development of an efficient discretization procedure}

\subsection{Optimization of the FORM approximation}

We present the optimal discretization for the FORM approximation $G_{L}(\mathbf{U})$ for $n=2$ and $n=3$ dimensions. Extension to higher numbers of random variables is discussed. Because the linear LSF employed in FORM is described only by the reliability index $\beta_{F O R M}$ and the vector $\boldsymbol{\alpha}$ of FORM sensitives (Eq. 4), it facilitates parametric studies.

Initially, we consider a reliability index $\beta_{F O R M}=4.26$, corresponding to a probability of failure of $10^{-5}$. The standard deviation of the additive measurement error is set to either $\sigma_{\varepsilon}=0.5$ or $\sigma_{\varepsilon}=1.0$. Different combinations of FORM sensitivity values $\alpha_{i}$ are selected, to investigate their effect on the optimal discretization. In all investigated cases, we find that the position of the midpoint of the optimal discretization frame coincides with the design point, i.e. $v_{i}^{o p t}=0$. The optimal discretization widths $w_{i}^{o p t}$ vary significantly with the importance measures $\alpha_{i}$, as shown in section 3.1.2. However, the optimal number of intervals $n_{i}^{o p t}$ is approximately the same for all random variables in all investigated cases, independent of the $\alpha_{i}$ values, i.e. $n_{i}^{o p t}=c_{u p}^{1 / n}$.

\subsubsection{On why the optimal number of intervals $n_{i}^{o p t}$ is independent of $\alpha_{i}$}

To better understand why the number of intervals does not depend on $\left|\alpha_{i}\right|$ (for $\left|\alpha_{i}\right|$ that are significantly larger than 0 ), recall that an efficient discretization scheme should focus on the area around the limit state surface. More precisely, the discretization error in the posterior 
case is induced by the cells that are cut by the limit state surface. Exemplarily, Fig. 8 shows a

360 linear problem in standard normal space with two basic random variables $U_{1}$ and $U_{2}$, where $361 \alpha_{1}=0.45$ and $\alpha_{2}=0.89$. While Fig. 8a shows an optimal discretization with 5 intervals per 362 dimension, Fig. 8 b shows a discretization scheme, where the more important random variable $363 U_{2}$ is discretized with 6 intervals and $U_{1}$ with 4 intervals. In both cases, the discretization 364 frame is centered at the design point. It is observed that the probability mass of the (grey) 365 cells, associated with the discretization error in the posterior case, is higher in Fig. 8b than for 366 the optimal discretion scheme in $8 \mathrm{a}$. In the example shown here it is $1.4 \cdot 10^{-3}$ compared to $367 \quad 2.5 \cdot 10^{-4}$.

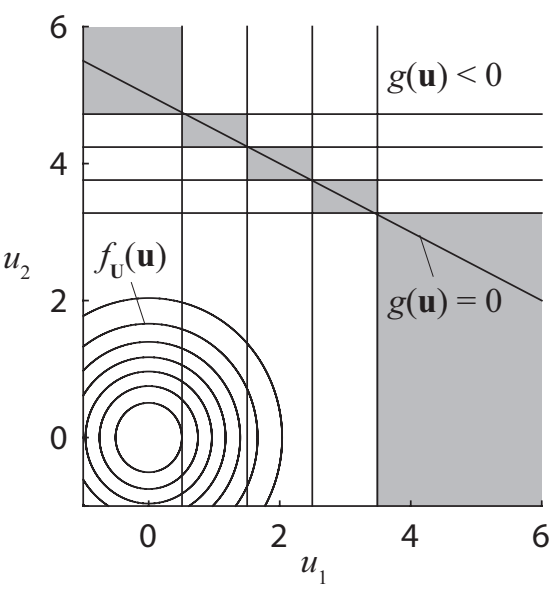

a) Optimal discretization

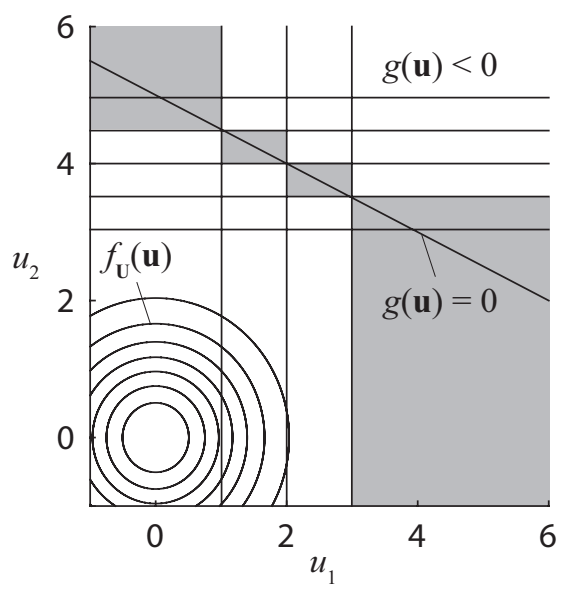

b) Suboptimal discretization

Figure 8. Linear problem in standard normal space, with two random variables $U_{1}$ and $U_{2}$, where $\alpha_{1}=0.45$ and $\alpha_{1}=0.89$. The intervals cut by the limit state surface, i.e. those which potentially lead to a posterior discretization error are marked in grey.

372 For input random variables $X_{i}$ with a value of $\left|\alpha_{i}\right|$ close to zero, the above observations do not hold. Following Section 2.4, these variables should be removed from the BN prior to discretization.

\subsubsection{Dependence of the optimal discretization width on $\alpha_{i}$}

In the optimization, it is found that the optimal discretization width $w_{i}^{o p t}$ varies strongly with the random variable's importance, expressed through $\alpha_{i}$. It is reminded that the width $w_{i}$ describes the domain in which a fine discretization mesh is applied (Fig. 6). In general, $w_{i}^{o p t}$ decreases with increasing $\alpha_{i}$. This effect can be observed in Fig. 8a, where $U_{2}$ is the more important input random variable and it is $w_{2}^{o p t}<w_{1}^{o p t}$.

381 A clear relation between $w_{i}^{\text {opt }}$ and $\alpha_{i}$ can be observed by plotting the probability mass enclosed by $w_{i}^{\text {opt }}$ against $\alpha_{i}$, as shown in Fig. 9. The results of Fig. 9 indicate that the probability mass contained within this interval should be a direct function of $\alpha_{i}$. The more important the variable, the finer the discretization around the design point should become. The observed relationship between this probability mass and $\alpha_{i}$ follows a clear trend, and a function can be fitted (Fig. 9). Neither the dimensionality of the problem nor the standard 
deviation of the measurement error appear to have an influence on this relation. However, as shown in the following section, it is found that the relation does depend on the prior failure probability of the problem (i.e. on $\beta_{F O R M}$ ) and on the number of intervals $n_{i}$ used to discretize the domain.

391

392

393

394

395

396

397

398

399

400

401

402

403

404

405

406

407

408

409

410

411

412

413

414

415

416

417

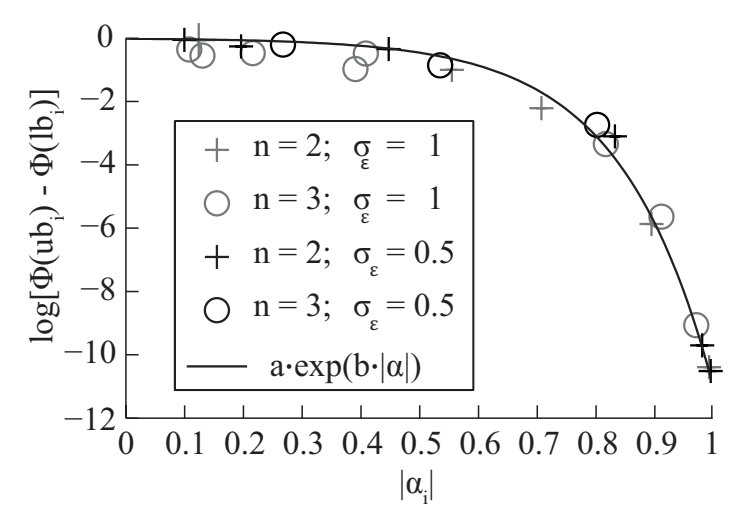

Figure 9. Logarithm of the probability mass enclosed by the discretization frame plotted against $\alpha_{i}$. $\Phi$ denotes the standard normal CDF and $u b_{i}$ respectively $l b_{i}$ the last (upper) and the first (lower) interval bound in dimension $i$.

To facilitate the application in practice and extending the results to larger numbers of random variables, in section 3.2 parametric functions are fitted to the optimization results to capture the dependency between the optimal discretization width $w_{i}^{\text {opt }}$ and the FORM importance measures $\alpha_{i}$.

\subsubsection{Dependence of the optimal discretization on the reliability index $\beta$ and the} number of discretization cells $c_{u p}$

The influence of the prior failure probability and the maximum size of the CPT, $c_{u p}$, on the optimal discretization is investigated through 10 problems with $n=2$ random variables in standard normal space. The FORM importance measures of the random variables are selected between 0.1 to 0.995 and the standard deviation of the measurement error is fixed to $\sigma_{\varepsilon}=1.0$. We find that the optimal discretization frame is generally centered at the design point, i.e. $v_{i}^{o p t}=0$, and that the intervals are distributed uniformly among the dimensions.

Firstly, we vary the maximum CPT size $c_{u p}$, i.e. the total number of discretization cells. The reliability index is $\beta_{F O R M}=5.2$. Fig. 10 shows the influence of $c_{u p}$ on the resulting width of the discretization frame $w_{i}$. Three cases are considered: $c_{u p}=25, c_{u p}=100$ and $c_{u p}=400$. These choices correspond to 5,10 and 20 intervals for each random variable. The left side of Fig. 10 shows the relation between the optimal $w_{i}$ and $\left|\alpha_{i}\right|$ The right side of Fig. 10 shows the same relation, where the $w_{i}$ 's are scaled as in Fig. 9, i.e. the logarithm of the probability mass enclosed by the outer interval boundaries is depicted. As in Fig. 9, there is a clear dependence between the scaled $w_{i}$ values and the $\left|\alpha_{i}\right|$ 's. The interval frames increase with increasing number of random variables.

Secondly, we vary the prior failure probability from $10^{-3}(\beta=3.1)$ to $10^{-7}(\beta=5.2)$. The results are shown in Fig. 11. Again, a distinct dependence between the scaled $w_{i}$ values and 
418 the $\left|\alpha_{i}\right|$ 's is found. The interval frames decrease with increasing reliability index (with 419 decreasing failure probability).

\subsection{Parametric function of optimal discretion frame}

421 As evident from Fig. 10 and Fig. 11, there is a clear dependence of the probability mass enclosed by the optimal discretization frame (with width $w_{i}$ ) on the FORM sensitivity values $\left|\alpha_{i}\right|$. The following parameteric function captures this dependence:

$\log \left(\Phi\left(u b_{i}\right)-\Phi\left(l b_{i}\right)\right)=a \cdot \exp \left(b \cdot\left|\alpha_{i}\right|\right)$

424

425

426

427

428

429

$u b_{i}$ is the upper and $l b_{i}$ the lower interval boundary in dimension $i$, such that $w_{i}=u b_{i}-l b_{i}$. $a$ and $b$ are the parameters of the exponential function. This function is depicted in Figs. 10 and 11. Tab. 1 shows the parameter values $a$ and $b$ for the different combinations of the prior reliability index $\beta$ and number of intervals per dimension

Table 1. Parameters $a$ and $b$ of Eq. 14 for $\beta=3.1, \beta=4.3$ and $\beta=5.2$ as well as 5,10 and 20 intervals per dimension.

\begin{tabular}{c|ccc}
{$\left[\begin{array}{l}a \\
b\end{array}\right]$} & $n_{i}=5$ & $n_{i}=10$ & $n_{i}=20$ \\
\hline$\beta=3.1$ & {$\left[\begin{array}{c}-0.28, \\
2.9\end{array}\right]$} & {$\left[\begin{array}{c}-1.6 \cdot 10^{-2} \\
5.8\end{array}\right]$} & {$\left[\begin{array}{c}-9.8 \cdot 10^{-4}, \\
8.7\end{array}\right]$} \\
$\beta=4.3$ & {$\left[\begin{array}{c}-0.15 \\
4.3\end{array}\right]$} & {$\left[\begin{array}{c}-2.4 \cdot 10^{-2} \\
6.1\end{array}\right]$} & {$\left[\begin{array}{c}-2.1 \cdot 10^{-2}, \\
6.2\end{array}\right]$} \\
$\beta=5.2$ & {$\left[\begin{array}{c}-0.36, \\
3.7\end{array}\right]$} & {$\left[\begin{array}{c}-0.11 \\
5.0\end{array}\right]$} & {$\left[\begin{array}{c}-3.7 \cdot 10^{-2} \\
6.0\end{array}\right]$}
\end{tabular}

430 From the left sides of Fig. 10 and Fig. 11, it can be observed that the relation between $\alpha_{i}{ }^{2}$ and 431 the optimal $w_{i}$ is fairly diffuse for random variables with $\left|\alpha_{i}\right|<0.6$. Here, the parametric 432 relationship of Eq. 14 is less accurate. However, these random variables by definition have 433 lower importance on the reliability estimate. Hence, the inaccuracy of Eq. 14 for random 434 variables with $\left|\alpha_{i}\right|<0.6$ is not critical, as is confirmed by the numerical investigations 435 performed in the remainder of the paper.

436 The parameter values of Tab. 1 are derived from two-dimensional problems. In Fig. 9 it is 437 shown that there are no notable differences between two and three dimensions. On this basis, 438 it is hypothesized that the heuristics are applicable also to problems with higher dimensions. 439 This assumption is furthermore supported by the verification examples presented in chapter 4 , 440 where the heuristics are applied also to four-dimensional problems without any notable 441 deterioration in the results. 
a) 5 intervals per random variable
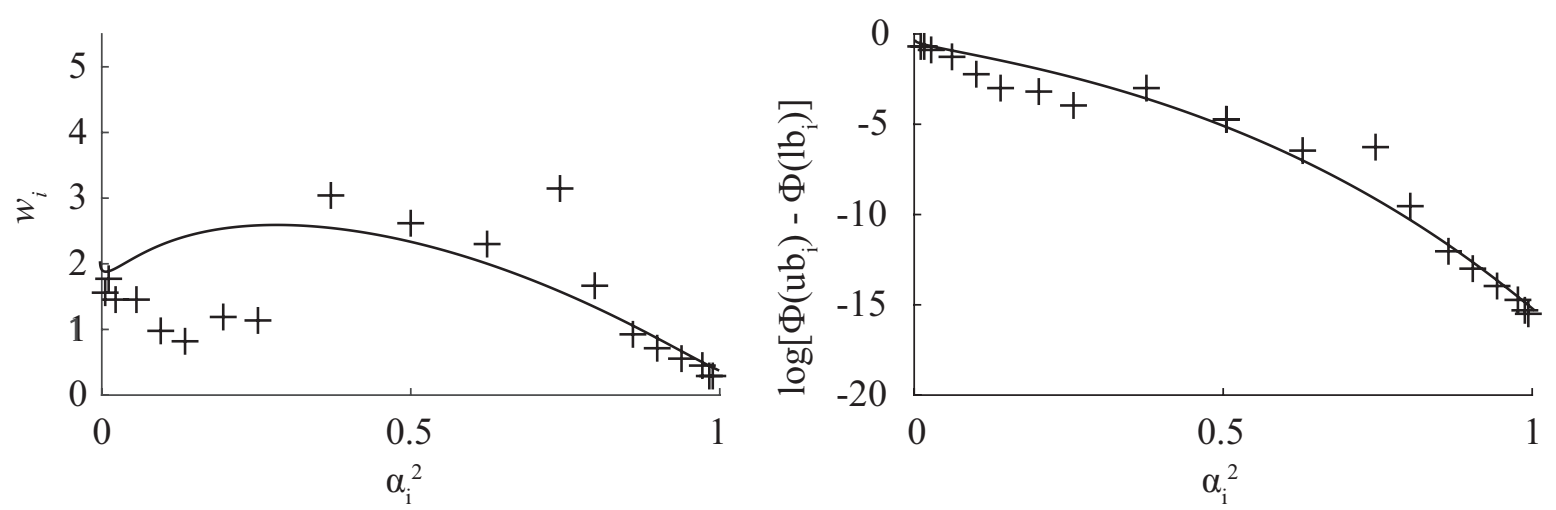

b) 10 intervals per random variable
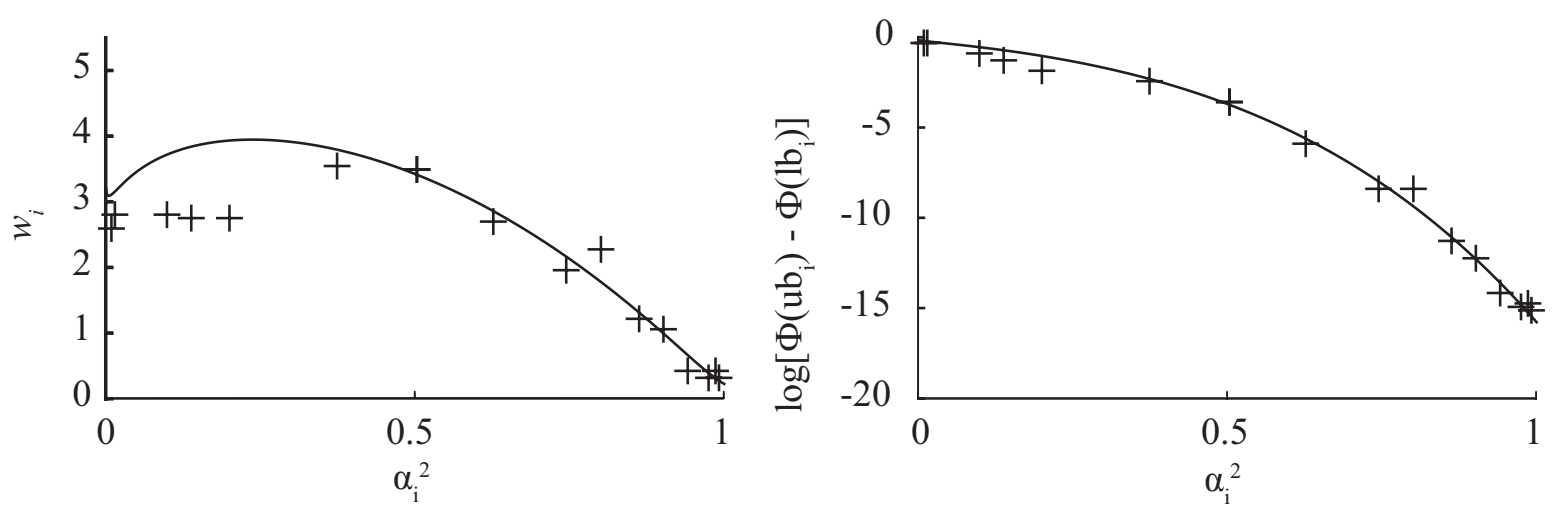

c) 20 intervals per random variable
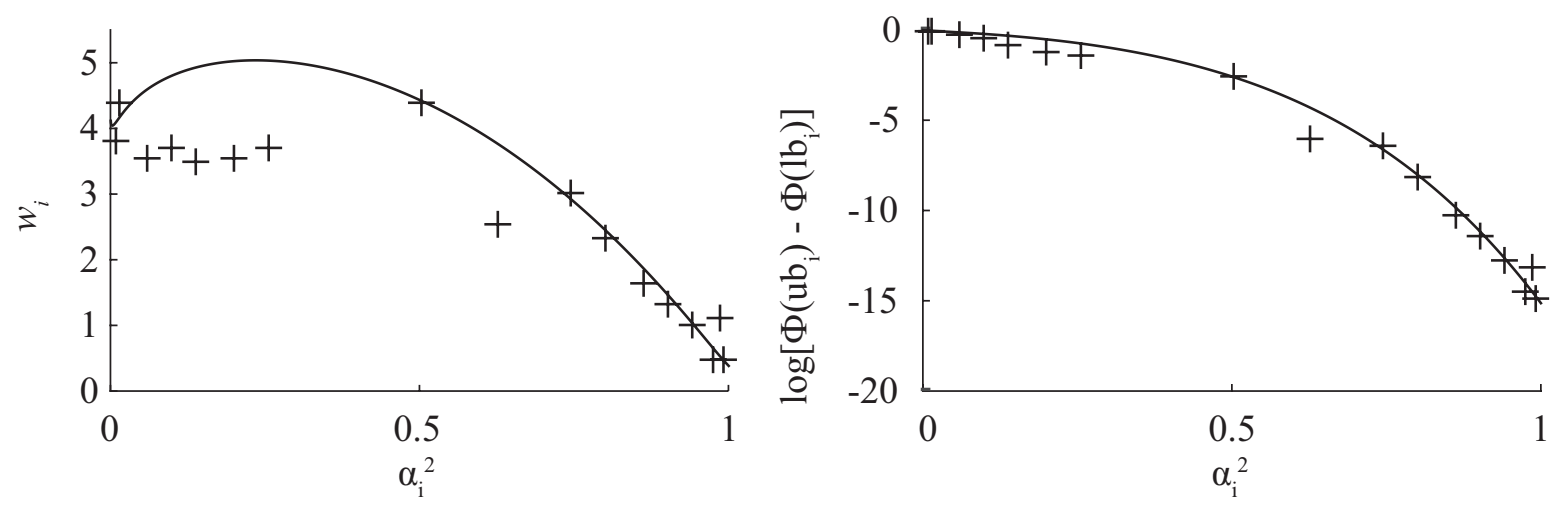

Figure 10. Optimization results for 10 two-dimensional, linear problems in standard normal space, which are discretized with 5, 10 and 20 intervals per dimension. In all cases the prior failure probability is $10^{-7}(\beta=5.2)$. The crosses represent the optimization results. The solid lines are the fitted parametric functions (Eq. 14). The left-hand side shows the relation between the width of a discretization frame $w_{i}$ and $\left|\alpha_{i}\right|$ and the right-hand side shows the relation between the probability mass enclosed by the discretization frame with width $w_{i}$ and $\left|\alpha_{i}\right|$. 
a) $\beta=3.1, \operatorname{Pr}(F)=10^{-3}$
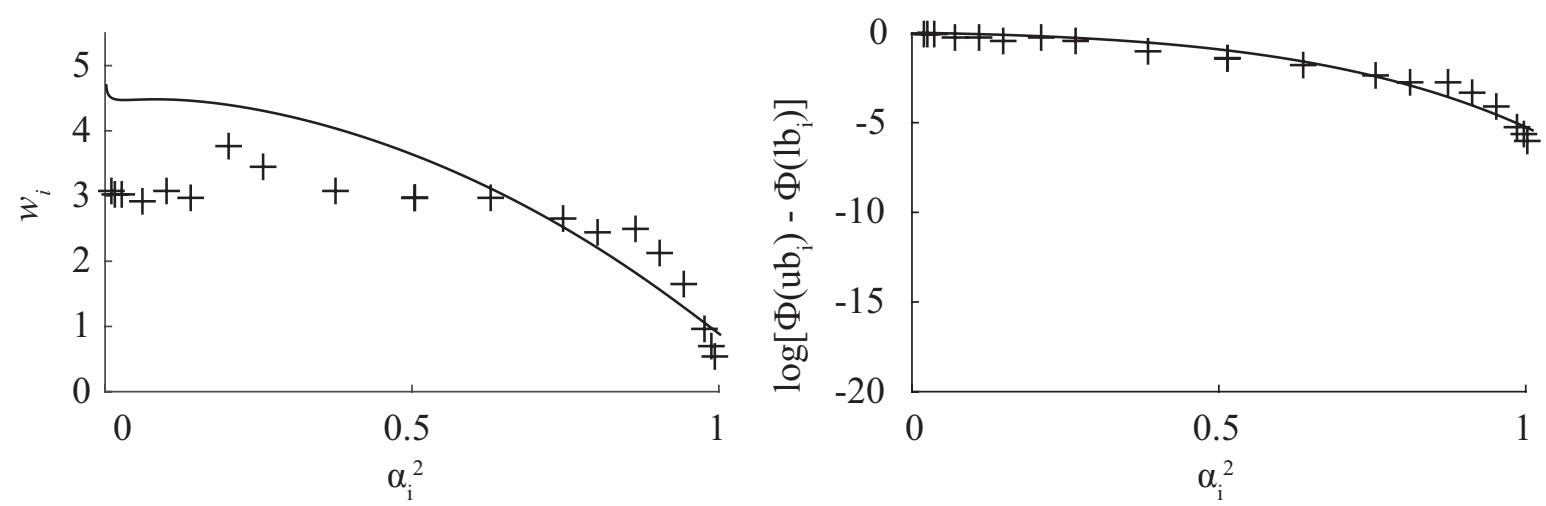

b) $\beta=4.3, \operatorname{Pr}(F)=10^{-5}$
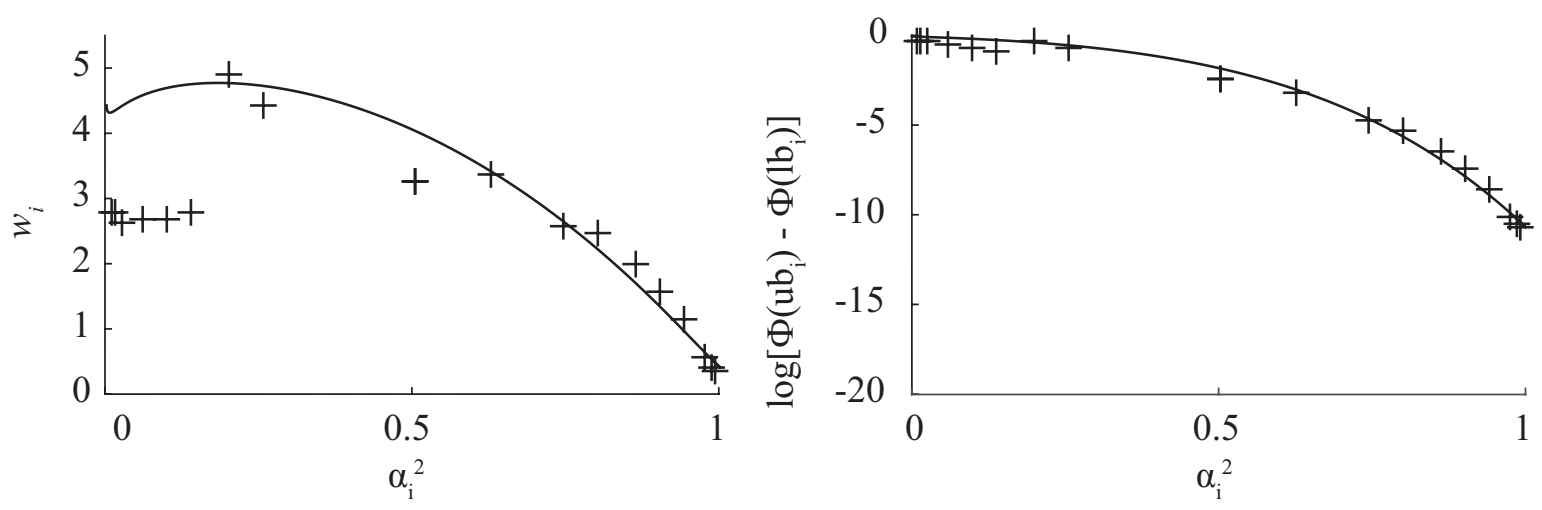

c) $\beta=5.2, \operatorname{Pr}(F)=10^{-7}$
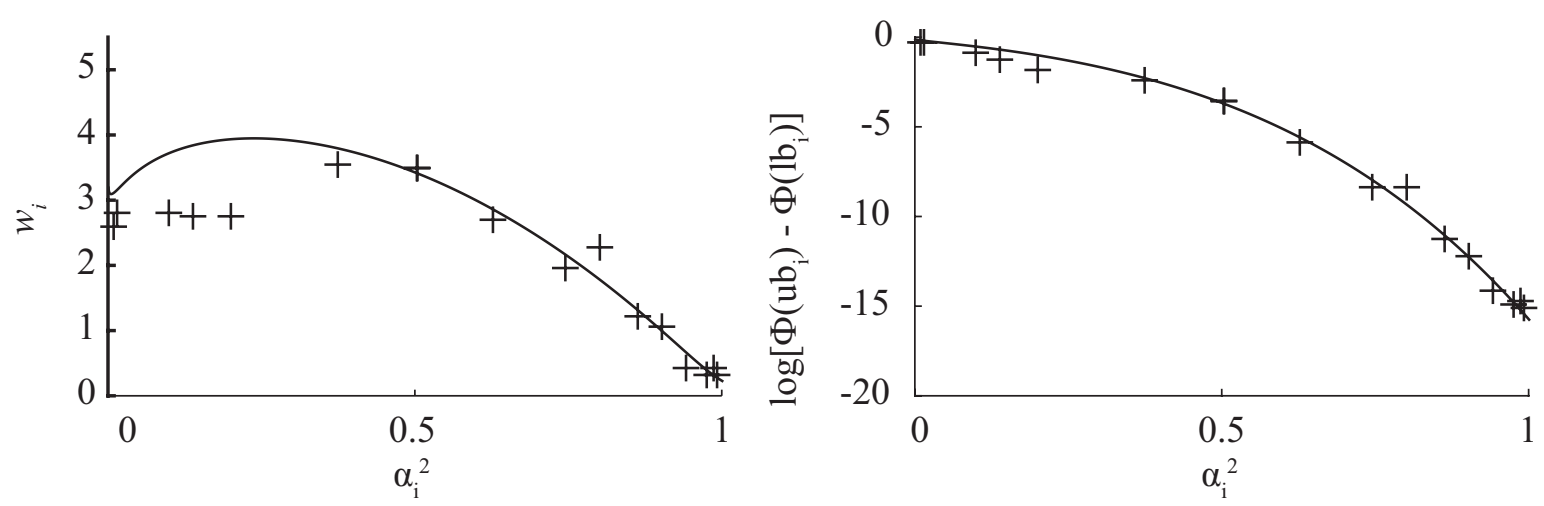

Figure 11. Optimization results for 10 two-dimensional, linear problems in standard normal space, which are discretized with 10 intervals per dimension. The prior failure probabilities are $10^{-3}(\beta=3.1), 10^{-5}(\beta=4.3)$ and $10^{-7}(\beta=5.2)$. The crosses represent the optimization results. The solid lines are the fitted parametric functions (Eq. 14). The left-hand side shows the relation between the width of a discretization frame $w_{i}$ and $\left|\alpha_{i}\right|$ and the right-hand side shows the relation between the probability mass enclosed by the discretization frame with width $w_{i}$ and $\left|\alpha_{i}\right|$.

\subsection{Summary of the proposed procedure}

456 The steps of the proposed procedure are:

1. Formulate the reliability problem 
2. Set up the corresponding BN

3. Perform a FORM analysis for the reliability problem

4. Simplify the BN by removing nodes based on:

a. their importance for prediction

b. their observability

c. whether or not a node simplifies modeling of dependencies

d. whether or not it is desired to explicitly show a node in the $\mathrm{BN}$ for communication purposes

5. Find the discretization scheme in U-space based on the proposed heuristics i.e.:

a. the discretization scheme is centered at the design point from the FORM analysis

b. the same number of intervals is used for each random variable

c. the width of the discretization frame follows Eq. 14

6. Transform the discretization scheme to X-space

7. Compute the CPTs of the component state node and the basic random variables using Monte Carlo simulation or Latin hypercube sampling

A MATLAB-based software tool performing these steps is available for download under www.era.bgu.tum.de/software.

\section{Applications}

\subsection{Verification example I}

For verification purposes, we apply the proposed methodology to the discretization of a general limit state with non-normal dependent random variables. The approximation error made by this discretization is investigated for different measurement outcomes.

Failure is defined through the $\operatorname{LSF} g(\mathbf{x})$ :

$g(\mathbf{x})=a-\prod_{i=1}^{n} X_{i}$

i.e., failure corresponds to the event $\left\{\prod_{i=1}^{n} X_{i} \geq a\right\}$.

The basic random variables are distributed as $X_{1} \sim \operatorname{LN}(0,0.5)$ and $X_{2}, \ldots, X_{n} \sim \operatorname{LN}(1,0.3)$ (values in parenthesis are the parameters of the lognormal distribution). The statistical dependence among the $X_{i}$ is described through a Gaussian copula model, with pairwise correlation coefficients $\rho_{i j}$. The parameters $a$ and $\rho_{i j}$ determine the prior failure probability $P_{F}$. Measurements $M_{\mathrm{i}}=m_{\mathrm{i}}$ are available for all basic random variables; they are associated with multiplicative measurement errors $\varepsilon_{\mathrm{i}} \sim \operatorname{LN}(0,0.71)$. In Tabs. 2 and 3 , different cases with 3 and 4 random variables are shown. These cases differ with respect to the prior failure 
probability $P_{F}$, the correlation between the random variables $\rho_{i j}$ and the observed measurements $\mathbf{m}$. For each case, a reference solutions $P_{F \mid \mathbf{M}}$ is calculated analytically.

Table 2. Evaluation of the discretization error for different measurement outcomes $\mathbf{m}$, for problems with $n=3$ random variables. $a$ is the constant in the LSF, Eq. 15; $\rho_{i j}$ is the correlation coefficient between $X_{i}$ and $X_{j}$ for all $i \neq j ; P_{F}$ and $\mathrm{P}_{\mathrm{F} \mid \mathbf{M}}$ denote the analytically calculated prior and posterior failure probabilities; $\hat{P}_{F \mid M}$ is the conditional failure probability calculated with the discrete $\mathrm{BN}$.

\begin{tabular}{ccccccccc}
\hline$a$ & $c_{u p}$ & $\rho_{i j}$ & $\mathrm{P}_{\mathrm{F}}$ & $\mathbf{m}$ & $\mathrm{P}_{\mathrm{F} \mid \boldsymbol{M}}$ & $\widehat{\mathrm{P}}_{\mathrm{F} \mid \boldsymbol{M}}$ & $\begin{array}{c}\text { Absolute } \\
\text { error }\end{array}$ & $\begin{array}{c}\text { Relative } \\
\text { error [\%] }\end{array}$ \\
\hline 100 & $10^{3}$ & 0 & $3.6 E-5$ & {$[3.0,2.9,2,9]$} & $4.3 E-5$ & $4.5 E-5$ & $3 E-6$ & 6 \\
100 & $10^{3}$ & 0 & $3.6 E-5$ & {$[2.3,1.1,2,1]$} & $4.6 E-6$ & $5.3 E-6$ & $7 E-7$ & 14 \\
100 & $10^{3}$ & 0 & $3.6 E-5$ & {$[0.9,2.4,0.9]$} & $2.8 E-7$ & $3.5 E-7$ & $7 E-8$ & 25 \\
200 & $15^{3}$ & 0.5 & $1.6 E-4$ & {$[1.6,2.0,1.2]$} & $1.4 E-6$ & $1.4 E-6$ & $1 E-7$ & 4 \\
400 & $8^{3}$ & 0.5 & $6.4 E-6$ & {$[2.6,3.0,3.2]$} & $8.2 E-7$ & $8.9 E-7$ & $7 E-8$ & 9 \\
400 & $12^{3}$ & 0.5 & $6.4 E-6$ & {$[3.6,3.3,4,3]$} & $4.9 E-6$ & $5.0 E-6$ & $1 E-9$ & 3 \\
\hline
\end{tabular}

Table 3. Evaluation of the discretization error for different measurement outcomes $\mathbf{m}$. The number of random variables $n=4 ; a$ is the constant in the LSF, Eq. 15; $\rho_{i j}$ is the correlation coefficient between $X_{i}$ and $X_{j}$ for all $i \neq j ; P_{F}$ and $P_{F \mid M}$ denote the analytically calculated prior and posterior failure probabilities; $\hat{P}_{F \mid M}$ is the conditional failure probability calculated with the discrete $\mathrm{BN}$.

\begin{tabular}{llllllllll}
$a$ & $c_{\text {up }}$ & $\rho_{i j}$ & $\mathrm{P}_{\mathrm{F}}$ & $\mathbf{m}$ & $\mathrm{P}_{\mathrm{F} \mid \boldsymbol{M}}$ & $\widehat{\mathrm{P}}_{\mathrm{F} \mid \boldsymbol{M}}$ & $\begin{array}{c}\text { Absolute } \\
\text { error }\end{array}$ & $\begin{array}{l}\text { Relative } \\
\text { error [\%] }\end{array}$ \\
\hline
\end{tabular}

\begin{tabular}{ccccccccc}
400 & $10^{4}$ & 0 & $1.7 E-5$ & {$[2.2,3.2,2.4,3.4]$} & $9.5 E-6$ & $1.0 E-5$ & $9 E-7$ & 9 \\
400 & $10^{4}$ & 0 & $1.7 E-5$ & {$[1.6,1.6,1.6,2.0]$} & $6.5 E-7$ & $7.9 E-7$ & $1 E-7$ & 21 \\
400 & $10^{4}$ & 0 & $1.7 E-5$ & {$[1.1,2.3,1.9,1.2]$} & $2.4 E-7$ & $3.0 E-7$ & $6 E-8$ & 26 \\
600 & $10^{4}$ & 0.5 & $1.3 E-3$ & {$[3.3,1.7,2.8,2.6]$} & $4.2 E-4$ & $4.3 E-4$ & $2 E-5$ & 4 \\
800 & $8^{4}$ & 0.5 & $5.3 E-4$ & {$[1.9,2.0,1.9,2.4]$} & $1.8 E-5$ & $1.9 E-5$ & $1 E-6$ & 8 \\
\hline
\end{tabular}

The results in Tables 2 and 3 show that the proposed methodology for discretization leads to errors in the posterior probability estimate that are acceptably small for most engineering applications. (It is reminded that discretization does not lead to a discretization error in the prior case.) As expected, the relative error is larger when the posterior probability is low, and the absolute error is larger when the posterior probability is high. This follows from the error measure defined in Eq. 11, which balances the relative with the absolute error. In addition, the results do not display any apparent effect of correlation on the accuracy. 
510 To assess the effect of the choice of the number of discretization intervals, the failure 511 probability $\widehat{\mathrm{P}}_{\mathrm{F} \mid \boldsymbol{M}}$ was calculated for a discretization scheme with up to 20 intervals per RV for 512 the fourth measurement case in Tab. 2. The estimated failure probabilities $\widehat{\mathrm{P}}_{\mathrm{F} \mid \boldsymbol{M}}$ are plotted 513 together with the exact solution in Fig. 12.

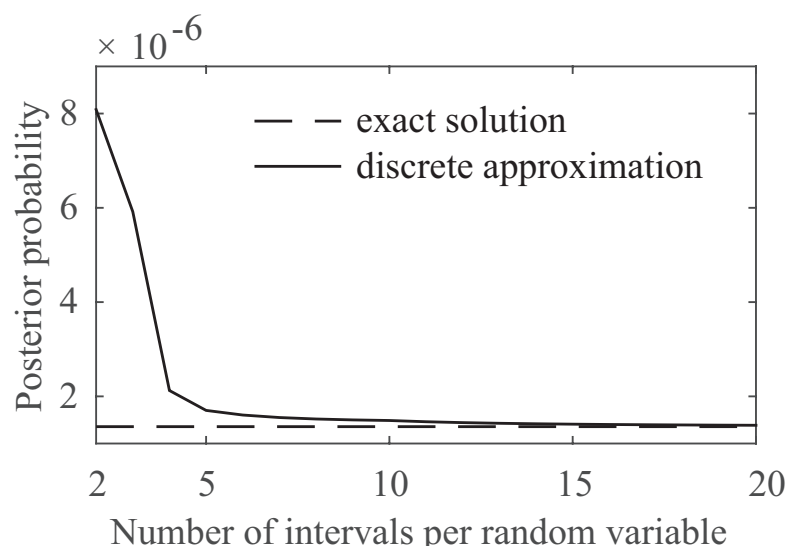

Figure 12. Posterior probability $\hat{P}_{F \mid M}$ as a function of the number of intervals per random variable together with the exact (analytical) solution $P_{F \mid M}$ for the fourth measurement case $[1.6,2.0,1.2]$ in Tab 2 .

\subsection{Verification example II}

518 The failure criterion applied in verification example I (Eq. 15) leads to a linear LSF in U519 space. To verify the accuracy of the proposed method for problems with non-linear LSFs in 520 U-space, we additionally investigate the following LSF:

$g(\mathbf{x})=a-\sum_{i=1}^{n} X_{i}$

521 Again the basic random variables $X_{1}$ to $X_{n}$ are distributed as $X_{1} \sim L N(0,0.5)$ 522 and $X_{2}, \ldots, X_{n} \sim \operatorname{LN}(1,0.3)$. Different cases with $n=2,3$ and 4 random variables are 523 investigated. Measurements $M_{\mathrm{i}}=m_{\mathrm{i}}$ are available for all basic random variables; associated 524 to these measurement are multiplicative measurement errors $\varepsilon_{\mathrm{i}} \sim \operatorname{LN}(0,0.71)$. For 525 independent random variables $X_{i}$ it is possible to determine posterior distributions $526 f_{X_{i} \mid M_{i}}\left(x_{i} \mid m_{i}\right)$ analytically. The posterior failure probabilities $P_{F \mid M}$, which are used as 527 reference solutions, are calculated through importance sampling with $10^{7}$ samples. The 528 results are presented in Tab. 4. 
Table 4. Evaluation of the discretization error for different measurement outcomes $\mathbf{m}$. The problems have

$530 n=2,3$ or 4 random variables; $a$ is the constant in the LSF, Eq. 16 ; $\rho_{i j}$ is the correlation coefficient between $X_{i}$

531 and $X_{j}$ for all $i \neq j ; P_{F}$ and $P_{F \mid M}$ denote the prior respectively posterior failure probabilities, which are calculated

532 through importance sampling with $10^{7}$ samples; $\hat{P}_{F \mid M}$ is the conditional failure probability calculated with the

533 discrete BN. Since for correlated basic random variables there is no analytical solution, in these cases the

534 updating of the basic random variables was performed through rejection sampling with $>5 E 7$ accepted samples.

\begin{tabular}{cccccccc}
\hline$a$ & $c_{u p}$ & $\rho_{i j}$ & $\mathrm{P}_{\mathrm{F}}$ & $\mathbf{m}$ & $\mathrm{P}_{\mathrm{F} \mid \boldsymbol{M}}$ & $\widehat{\mathrm{P}}_{\mathrm{F} \mid \boldsymbol{M}}$ & $\begin{array}{c}\text { Absolute } \\
\text { error }\end{array}$ \\
& & $\begin{array}{c}\text { Rel. } \\
\text { error } \\
{[\%]}\end{array}$ \\
\hline
\end{tabular}

\begin{tabular}{|c|c|c|c|c|c|c|c|c|}
\hline 12 & $10^{2}$ & 0 & $1.3 E-5$ & {$[2.8,4.5]$} & $1.4 E-5$ & $1.2 E-5$ & $2 E-6$ & 15 \\
\hline 12 & $10^{2}$ & 0 & $1.3 E-5$ & {$[2.3,2.4]$} & $3.3 E-6$ & $3.5 E-6$ & $2 E-7$ & 6 \\
\hline 10 & $12^{2}$ & 0 & $1.7 E-4$ & {$[4.0,3.2]$} & $4.0 E-4$ & $3.7 E-4$ & $3 E-5$ & 7 \\
\hline 12 & $10^{2}$ & 0.5 & $1.7 E-4$ & {$[2.3,2.4]$} & $4.8 E-5$ & $5.0 E-5$ & $2 E-6$ & 4 \\
\hline \multicolumn{9}{|c|}{$\underline{n=3:}$} \\
\hline 15 & $10^{3}$ & 0 & $3.7 E-5$ & {$[2.1,5.6,5.0]$} & $4.8 E-5$ & $4.5 E-5$ & $4 E-6$ & 7 \\
\hline 15 & $10^{3}$ & 0 & $3.7 E-5$ & {$[1.1,3.7,3.4]$} & $1.5 E-5$ & $1.8 E-5$ & $3 E-6$ & 20 \\
\hline 13 & $12^{3}$ & 0 & $5.0 E-4$ & {$[3.0,3.0,3.0]$} & $5.4 E-4$ & $5.4 E-5$ & $3 E-6$ & 1 \\
\hline 16 & $10^{3}$ & 0.5 & $9.1 E-4$ & {$[3.0,6.0,5.0]$} & $1.8 E-3$ & $1.9 E-3$ & $3 E-5$ & 2 \\
\hline \multicolumn{9}{|c|}{$\underline{n=4:}$} \\
\hline 20 & $8^{4}$ & 0 & $7.4 E-6$ & {$[2.0,4.0,3.4,3.0]$} & $4.9 E-6$ & $5.6 E-6$ & $6 E-7$ & 13 \\
\hline 17 & $8^{4}$ & 0 & $3.1 E-4$ & {$[1.0,1.4,1.2,2.0]$} & $4.5 E-5$ & $5.2 E-5$ & $7 E-6$ & 15 \\
\hline 17 & $12^{4}$ & 0 & $3.1 E-4$ & {$[3.1,2.0,3.3,2.4]$} & $2.5 E-4$ & $2.5 E-4$ & $7 E-6$ & 3 \\
\hline 24 & $8^{4}$ & 0.5 & $3.7 E-4$ & {$[1.0,1.4,1.2,2.0]$} & $1.1 E-5$ & $1.1 E-5$ & $9 E-7$ & 8 \\
\hline
\end{tabular}

537 The results in Tab. 4 do not differ substantially from Tabs. 2 and 3. This indicates that the 538 (weak) non-linearity of the LSF function describing failure does not affect the accuracy 539 significantly.

\section{$540 \quad 4.3$ Runway overrun}

541 Runway overrun (RWO) of a landing aircraft is one of the most critical accidents types in 542 civil aviation (IATA, 2013). A conceptual RWO warning system is developed with the 
proposed discretization procedure. It provides RWO probabilities conditional on observations

544 of the landing-weight, the headwind and the approach speed for different aircraft types and 545 different airports. For a detailed description of how this problem can be treated in BN 546 framework we refer to (Zwirglmaier and Straub, 2015).

547 RWO is the event of the operational landing distance exceeding the available runway length 548 (Fig. 13). Correspondingly, a LSF for runway overrun can be defined as:

$$
g(\mathbf{X})=\text { Runway length }- \text { Operational landing distance }(\mathbf{X})
$$

549 with $\mathbf{X}$ representing the basic random variables of the problem.

550 (Drees and Holzapfel, 2012) proposed a model for the operational landing distance required 551 by a landing aircraft, which is applied here. The model, as well as the basic random variables $552 \mathbf{X}$, are presented in (Zwirglmaier et al., 2014), which also includes a detailed description of 553 the reliability and sensitivity analysis.

554 We consider two different airports (AP I and AP II) and two different aircraft types (AC A 555 and $\mathrm{AC}$ B). While the aircraft type affects the landing-weight, the airport affects both the 556 headwind and the approach speed. The distribution models for landing-weight, headwind and 557 approach speed deviation at the different airports and with the different aircraft types are 558 given in Tabs. 5-7. All other basic random variables of the problem are not affected by the 559 airport and aircraft type and are as in (Zwirglmaier et al., 2014).

560 Tab. 8 summarizes the FORM importance measures of all random variables $\mathbf{X}$ computed for 561 the four combinations of aircrafts and airports.

562
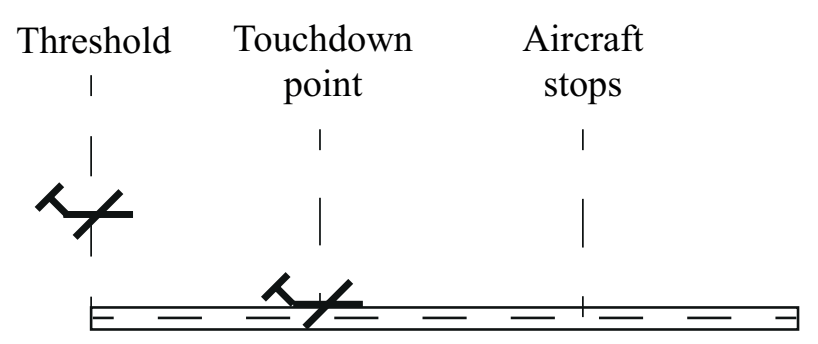

Runway length

Figure 13. Runway definitions. 
Table 5. Distribution models for landing weight conditional on the aircraft.

Landing weight $[\mathrm{t}]$

\begin{tabular}{cccc} 
Aircraft & Distribution & Mean & Std. deviation \\
\hline A & Weibull (min) & 59.25 & 1.69 \\
B & Weibull (min) & 64.25 & 1.69 \\
\hline
\end{tabular}

569 Table 6. Distribution models for head wind conditional on the airport.

$$
\text { Head wind [kts] }
$$

\begin{tabular}{crrc} 
Airport & Distribution & Mean & Std. deviation \\
\hline I & Normal & 5.42 & 5.75 \\
II & Normal & 6.51 & 5.75 \\
\hline
\end{tabular}

570

Table 7. Distribution models for approach speed deviation conditional on the airport.

\begin{tabular}{crcc} 
Airport & Distribution & Mean & Std. deviation \\
\hline I & Gumbel (max) & 4.69 & 4.21 \\
II & Gumbel (max) & 5.63 & 4.21 \\
\hline
\end{tabular}

Table 8. FORM importance measures $\alpha_{i}$ for each aircraft-airport combination and every basic random variable 575 in the RWO application.

\begin{tabular}{lccccc}
\hline & \multicolumn{3}{c}{$\alpha_{i}$} & \\
\cline { 2 - 4 } Random variable & $(\mathrm{I} / \mathrm{A})$ & $(\mathrm{I} / \mathrm{B})$ & $(\mathrm{II} / \mathrm{A})$ & $(\mathrm{II} / \mathrm{B})$ & Annotation \\
\hline Landing weight [t] & 0.09 & 0.10 & 0.11 & 0.09 & Modeled \\
Headwind [kts] & -0.65 & -0.61 & -0.67 & -0.60 & Modeled \\
Temperature [ $\left.{ }^{\circ} \mathrm{C}\right]$ & 0.03 & -0.00 & -0.03 & -0.03 & Not important \\
Air pressure [hPa] & 0.01 & -0.01 & -0.01 & -0.00 & Not important \\
Touchdown point [m] & 0.20 & 0.16 & 0.18 & 0.20 & Modeled \\
Approach speed deviation [kts] & 0.20 & 0.21 & 0.20 & 0.24 & Not observable \\
Time of spoiler deployment [s] & -0.00 & -0.00 & 0.01 & 0.01 & Not important \\
Time of breaking initiation [s] & 0.70 & 0.74 & 0.68 & 0.73 & Not observable \\
Time of reverser deployment [s] & 0.03 & 0.04 & 0.06 & 0.05 & Not important \\
Time of breaking end [s] & -0.02 & -0.01 & 0.01 & 0.02 & Not important
\end{tabular}




\subsubsection{Selection of relevant random variables}

577 The applied RWO model includes 10 basic random variables. However, it is sufficient to 578 include only a selection of these explicitly in the BN. Random variables that are not relevant 579 for the prediction of RWO in the considered scenarios can be excluded. This is the case for 580 random variables with a low FORM importance, whose value does not depend significantly 581 on airport and aircraft type. Here, all random variables, whose absolute value of the FORM 582 importance measure $\left|\alpha_{i}\right|^{\prime} s$ is smaller than 0.1 , are excluded (see Tab. 8). The one exception is 583 landing weight, since its mean value is substantially influenced by the aircraft type.

584 One can additionally exclude random variables that cannot be measured before the decision 585 on whether to land or not is made. This holds for Touchdown point and the time at which the 586 pilot initiates breaking. Since these basic random variables are also not needed to simplify the 587 modeling of dependencies, it is not necessary to explicitly model them in the BN, as indicated 588 in Tab. 8.

589

590

591 592

593

594

595

596

597

598

599

600

601

$$
m_{i}=x_{i}+\varepsilon_{i}
$$

602

603

604

605

606

607

608

609

610 BN. error:

\subsubsection{BN model}

The resulting BN of the RWO warning system is shown in Fig. 14. During the aircraft approach, measurements can be obtained for the three basic random variables included in the

The random variables are discretized separately for each aircraft-airport combination (joint states of discrete parents) with 8 intervals each, following the proposed discretization procedure. In a second step, the discretization schemes are merged, i.e. the regions of the outcome space, which are discretized with fine intervals for at least one of the aircraft-airport combinations, are discretized with the respective fine intervals also in the merged discretization scheme. In the end 15 (landing-weight), 10 (headwind) and 9 (approach speed deviation) intervals are used to discretize the three basic random variables.

For all observable quantities, the measurements $m_{i}$ are modeled with an additive observation

$\varepsilon_{i}$ is modeled by a normal distribution with zero mean and standard deviation $\sigma_{\varepsilon_{i}}$.

For the random variable landing weight (at landing time), the standard deviation of the measurement error is $\sigma_{\varepsilon_{L W}}=0.34$ tons. Due to turbulences governing wind speeds, the measurement of the head wind speed at the time of the measurement is only an uncertain indicator for the head wind speed at landing time; we model the measurement error with a standard deviation $\sigma_{\varepsilon_{H W}}=2.88 \mathrm{kts}$. The measurement uncertainty associated with the approach speed deviation at landing has standard deviation $\sigma_{\varepsilon_{A S D}}=4.21 \mathrm{kts}$.

49 (Measurement LW), 57 (Measurement HW) and 57 (Measurement ASD) intervals are used to discretize the measurement nodes. 


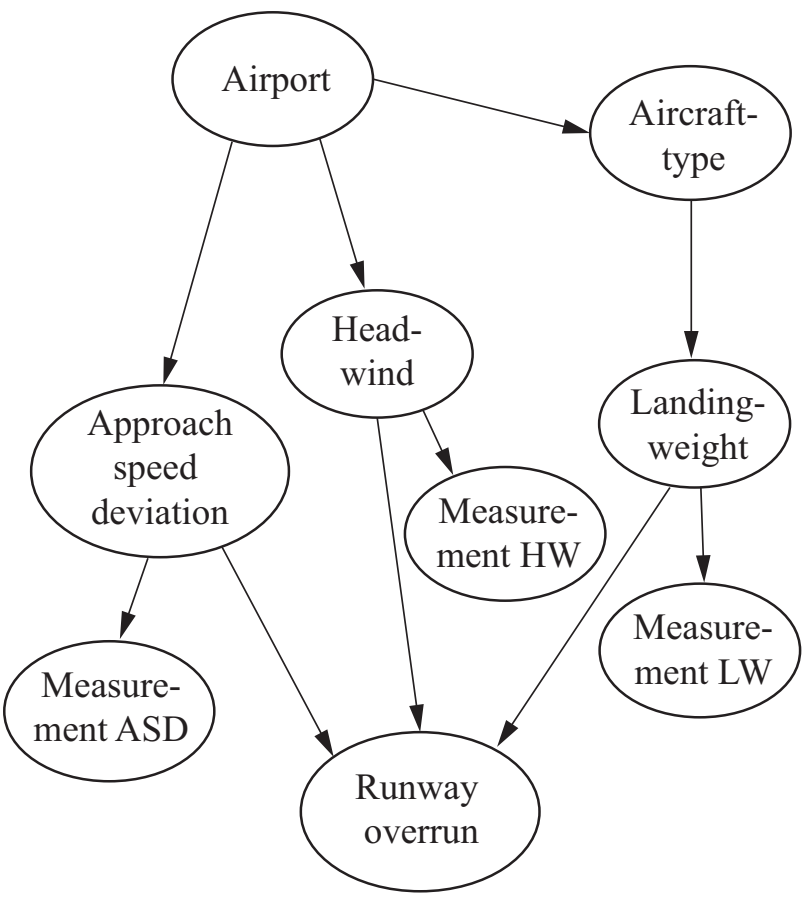

613 Figure 14. BN structure for a RWO warning system.

\section{$614 \quad 4.3 .3$ Results}

615 In Tab. 9, RWO probabilities for the different airports and aircrafts obtained with the discrete $616 \mathrm{BN}$ are compared to solutions, which were calculated by importance sampling around the 617 design point.

618 Table 9. RWO probabilities for the different airports and aircrafts calculated with the discrete $\mathrm{BN} p_{B N^{\prime}}$ 619 together with solutions calculated by importance sampling around the design point $p_{D S}$. The latter have a 620 sampling error with coefficient of variation in the order of $10 \%$.

621

\begin{tabular}{ccc}
\hline $\mathrm{AP} / \mathrm{AC}$ & $p_{B N}$ & $p_{D S}$ \\
\hline $\mathrm{I} / \mathrm{A}$ & $2.0 e-7$ & $1.9 e-7$ \\
$\mathrm{I} / \mathrm{B}$ & $1.0 e-6$ & $9.2 e-7$ \\
$\mathrm{II} / \mathrm{A}$ & $1.3 e-7$ & $1.3 e-7$ \\
$\mathrm{II} / \mathrm{B}$ & $6.9 e-7$ & $6.5 e-7$ \\
\hline
\end{tabular}

622 In Tab. 10, results obtained with the $\mathrm{BN}$ for different hypothetical cases of aircrafts 623 approaching an airport are presented. In each of these cases, measurements associated with 624 landing weight, headwind and the approach speed deviation are made. A threshold on the 625 probability of RWO is used to decide, whether or not the pilot should continue landing or 626 cancel the landing attempt. Here we assume that up to a RWO probability of $10^{-6}$ the pilot 627 should continue landing.

628 Table 10. Probabilities of RWO and corresponding decision on landing, computed with the BN for different 629 sets of observations. 


Case Airport Aircraft Meas. LW $\quad$ Meas. HW $\quad$ Meas. ASD [kts] $\quad$ Pr (RWO) $\quad$ Landing

\begin{tabular}{|c|c|c|c|c|c|c|c|}
\hline a) & I & B & 63 & 0 & 10.5 & $2.5 e-8$ & Yes \\
\hline b) & I & A & 61 & -10 & 5 & $4.8 e-6$ & No \\
\hline c) & II & B & 67 & 3 & 0 & $6.5 e-10$ & Yes \\
\hline d) & II & A & 57.5 & -12 & 3 & $1.3 e-6$ & No \\
\hline
\end{tabular}

\section{Discussion}

When modeling with BNs, it is often necessary or beneficial to discretize continuous random variables. When the $\mathrm{BN}$ includes rare events that are a function of such random variables, the choice of the discretization scheme is non-trivial. In this contribution, we investigate this discretization based on FORM concepts, and propose a heuristic procedure for an efficient discretization in these cases. This is based on importance measures $\alpha_{i}$ obtained through a FORM analysis, which represent the influence of the uncertainty associated with a random variable $X_{i}$.

638 The most important finding is that discretization should focus on the area around the most 639 likely failure point (design point), identified by a FORM analysis. Furthermore, we find that optimally all random variables should be discretized with approximately equal numbers of intervals, independent of their importance, as long as $\left|\alpha_{i}\right|$ is not close to zero. The widths of the intervals should be selected based on the FORM importance $\alpha_{i}$ of the random variables. With increasing importance, the interval width should be reduced, leading to finer discretization for larger $\left|\alpha_{i}\right|$. This relation is particularly evident for $\left|\alpha_{i}\right| \geq 0.8$. We show that it is possible to fit a parametric function to approximate the relation between $\left|\alpha_{i}\right|$ and the optimal width of the region on which the discretization should focus.

647 This parametric function is used to derive a heuristic procedure for finding an efficient 648 discretization. This allows the extrapolation of the optimization results to problems with more random variables. As demonstrated by the verification examples, the heuristic procedure leads

650 to accurate results.

651 This paper is restricted to static discretization. Application of the proposed procedure within 652 dynamic discretization (e.g. (Neil et al., 2008)) should be investigated. The results of the 653 procedure can serve as an initial discretization scheme, which is iteratively adjusted within 654 dynamic discretization. This might strongly enhance the convergence performance of these 655 algorithms.

656 The gain in computational efficiency resulting from the proposed procedure over alternative 657 static discretization approaches is problem specific. Some insights can be gained from Figure 658 8. A discretization with intervals of equal width centered at the origin would require between 659 approximately 2 to 5 times more intervals per random variable to achieve the same accuracy. 
660 Because of the exponential increase of computational effort with number of random variables, 661 this leads to a considerable increase in efficiency. The gain compared to discretization with 662 equal-frequency intervals is expected to be even higher, since equal frequency intervals 663 focuses the fine intervals on the region of high probability density rather than on the tails of 664 the distribution.

665 This paper focuses on component reliability problems, which are characterized by a single 666 design point. Nevertheless the heuristics derived can also be applied to system reliability 667 problems. System reliability problems can in general be treated as combinations of 668 component reliability problems. Parallel and series systems are to be distinguished. For 669 parallel systems discretization should be performed based on the joint design point of the 670 problem. For series systems, following the same line of thought as in the runway overrun 671 example, discretization can be performed separately for each component problem 672 (corresponds to the discrete cases i.e. airport- aircraft combinations in the RWO example). In 673 a second step the discretization schemes can be merged. In the same way it is possible to 674 apply the heuristic to multi state components. One can treat each limit state surface (LSF) 675 defining the boundary between two states separately and merge the discretization schemes 676 afterwards.

677 The number of basic random variables in a single LSF that can be modeled explicitly in a BN 678 is limited to around 5 to 8 . This is due to the exponential growth of the target nodes CPT with 679 increasing number of parents and is independent of the discretization method. Despite this 680 limitation, BNs are applicable to many practical problems - particularly if one considers that 681 usually not all basic random variables need to be modeled explicitly as nodes, as 682 demonstrated in the presented example.

683 While in this paper the focus was on the discretization of the basic random variables, it is 684 straightforward to incorporate the BNs discussed into larger models.

\section{Conclusion}

686 We investigate discretization of continuous reliability problems such that they can be treated 687 in a discrete Bayesian network framework. Reliability problems with linear LSF in standard 688 normal space are considered. These can be seen as FORM approximations of reliability 689 problems. For these linear LSFs, optimal discretization schemes are found, which are optimal 690 with respect to an error measure calculated through a preposterior analysis. Since FORM is

691 known to give good approximations also for most non-linear reliability problem, the resulting 692 discretization schemes are efficient also for non-linear LSFs. The main findings presented in 693 this paper are:

694 - An optimal discretization scheme should discretize finely the area around the FORM 695 design point. 
- The size of the sub-region of the outcome space of a random variable $X_{i}$ can be reduced significantly for random variables whose corresponding uncertainty is dominating the reliability problem

- The number of intervals used for discretization should be approximately equal for all basic random variables

On this basis, we propose a heuristic that can be used to find an efficient discretization scheme. In verification examples, this heuristic is found to give good accuracy and efficiency.

\section{References}

AGENA. 2005. Available: http://www.agenarisk.com.

AU, S.-K. \& BECK, J. L. 2001. Estimation of small failure probabilities in high dimensions by subset simulation. Probabilistic Engineering Mechanics, 16, 263-277.

DER KIUREGHIAN, A. 2005. First- and Second-Order Reliability Methods. In: NIKOLAIDIS, E., GHIOCEL, D. M. \& SINGHAL, S. (eds.) Engineering Design Reliability: Handbook. CRC PressINC.

DITLEVSEN, O. \& MADSEN, H. O. 2007. Structural Reliability Methods. John Wiley \& Sons.

DOUGHERTY, J., KOHAVI, R. \& MEHRAN, S. Supervised and Unsupervised Discretization of Continuous Features. In: PRIEDITIS, A. \& RUSSEL, S., eds. Machine Learning: Proceedings of the Twelfth International Conference, 1995 San Francisco.

DREES, L. \& HOLZAPFEL, F. 2012. Determining and Quantifying Hazard Chains and their Contribution to Incident Probabilities in Flight Operation. AIAA Modeling and Simulation Technologies Conference. American Institute of Aeronautics and Astronautics.

FRIIS-HANSEN, A. 2000. Bayesian Networks as a Decision Support Tool in Marine Applications. $\mathrm{PhD}$, Technical university of denmark.

HANEA, A., NAPOLES, O. M. \& ABABEI, D. 2015. Non-parametric Bayesian networks: Improving theory and reviewing applications. Reliability Engineering \& System Safety, 144, 265-284.

HANEA, A. M., KUROWICKA, D. \& COOKE, R. M. 2006. Hybrid method for quantifying and analyzing Bayesian belief nets. Quality and Reliability Engineering International, 22, 709-729.

HOHENBICHLER, M. \& RACKWITZ, R. 1981. Nonnormal dependent vectors in structural safety. Journal of Engineering Mechanics, 107, 1227-1238.

IATA 2013. Safety report 2012. International Air Transport Association (IATA).

JENSEN, F. V., LAURITZEN, S. L. \& OLESEN, K. G. 1990. Bayesian updating in causal probabilistic networks by local computations. Computational statistics quarterly, 4, 269-282.

JENSEN, F. V. \& NIELSEN, T. D. 2007. Bayesian networks and decision graphs, New York [u.a.], Springer.

KJAERULFF, U. B. \& MADSEN, A. L. 2013. Bayesian Networks and Influence Diagrams: A Guide to Construction and Analysis, Springer Publishing Company, Incorporated.

KOTSIANTIS, S. \& KANELlOPOULOS, D. 2006. Discretization techniques: A recent survey. GESTS International Transactions on Computer Science and Engineering, 32, 47-58.

KOZLOV, A. V. \& KOLLER, D. 1997. Nonuniform dynamic discretization in hybrid networks. 13th Annual Conference on Uncertainty in AI (UAI). Providence, Rhode Island.

LANGSETH, H., NIELSEN, T. D., RUMI, R. \& SALMERÓN, A. 2012. Mixtures of truncated basis functions. International Journal of Approximate Reasoning, 53, 212-227.

LANGSETH, H., NIELSEN, T. D., RUMÍ, R. \& SALMERÓN, A. 2009. Inference in hybrid Bayesian networks. Reliability Engineering \& System Safety, 94, 1499-1509. 
LAURITZEN, S. L. \& SPIEGELHALTER, D. J. 1988. Local computations with probabilities on graphical structures and their application to expert systems. Journal of the Royal Statistical Society. Series B (Methodological), 157-224.

LERNER, U. N. 2002. Hybrid Bayesian networks for reasoning about complex systems. PhD, Stanford University.

NEIL, M., TAILOR, M., MARQUEZ, D., FENTON, N. \& HEARTY, P. 2008. Modelling dependable systems using hybrid Bayesian networks. Reliability Engineering \& System Safety, 93, 933939.

RACKWITZ, R. 2001. Reliability analysis - a review and some perspectives. Structural Safety, 23, 365-395.

RAIFFA, H. \& SCHLAIFER, R. 1961. Applied statistical decision theory, Boston, Division of Research, Graduate School of Business Adminitration, Harvard University.

SINDEL, R. \& RACKWITZ, R. 1998. Problems and Solution Strategies in Reliability Updating. Journal of Offshore Mechanics and Arctic Engineering, 120, 109-114.

STRAUB, D. 2009. Stochastic Modeling of Deterioration Processes through Dynamic Bayesian Networks. Journal of Engineering Mechanics, 135, 1089-1099.

STRAUB, D. 2011. Reliability updating with equality information. Probabilistic Engineering Mechanics, 26, 254-258.

STRAUB, D. 2014a. Engineering Risk Assessment. Risk - A Multidisciplinary Introduction. Springer.

STRAUB, D. 2014b. Value of information analysis with structural reliability methods. Structural Safety, 49, 75-85.

STRAUB, D. \& DER KIUREGHIAN, A. 2010a. Bayesian Network Enhanced with Structural Reliability Methods: Application. Journal of Engineering Mechanics, 136, 1259-1270.

STRAUB, D. \& DER KIUREGHIAN, A. 2010b. Bayesian Network Enhanced with Structural Reliability Methods: Methodology. Journal of Engineering Mechanics, 136, 1248-1258.

STRAUB, D., PAPAIOANNOU, I. \& BETZ, W. 2016. Bayesian analysis of rare events. Accepted for publication in Journal of Computational Physics.

ZHANG, N. L. \& POOLE, D. A simple approach to Bayesian network computations. Proc. of the Tenth Canadian Conference on Artificial Intelligence, 1994.

ZHU, J. \& COLLETTE, M. 2015. A dynamic discretization method for reliability inference in Dynamic Bayesian Networks. Reliability Engineering \& System Safety, 138, 242-252.

ZWIRGLMAIER, K., DREES, L., HOLZAPFEL, F. \& STRAUB, D. 2014. Reliability analysis for runway overrun using subset simulation. ESREL 2014. Wroclaw, Poland.

ZWIRGLMAIER, K. \& STRAUB, D. 2015. Discretization of Structural Reliability Problems:An Application to Runway Overrun. 12th International Conference on Applications of Statistics and Probability in Civil Engineering, ICASP12. Vancouver, Canada. 